\title{
THE SPECTRA OF RANDOM PSEUDO-DIFFERENTIAL OPERATORS
}

\author{
JINGBO XIA
}

\begin{abstract}
We study the spectra of random pseudo-differential operators generated by the same symbol function on different $L^{2}$-spaces. Our results generalize the spectral coincidence theorem of S. Kozlov and M. Shubin (Math. USSRSb. 51 (1985), 455-471) for elliptic operators of positive order associated with ergodic systems. Because of our new approach, we are able to treat operators of arbitrary order and associated with arbitrary dynamical systems. Furthermore, we characterize the spectra of these operators in terms of certain naturally obtained Borel measures on $\mathbf{R}$.
\end{abstract}

\section{INTRODUCTION}

The purpose of this paper is to compare the spectra of pseudo-differential operators on different $L^{2}$-spaces induced by the same symbol function. The central question here is that do these operators have the same spectrum because of their common symbol function? To illustrate what we will do, let us consider the following problem. Suppose that we have an $\mathbf{R}^{n}$-flow on a probability space $(\Omega, \mathscr{B}, \mu)$, i.e., a strongly continuous measure-preserving map $(\omega, t) \mapsto \omega+t$ from $\Omega \times \mathbf{R}^{n}$ to $\Omega$. A differential expression

$$
\sum_{\alpha} a_{\alpha} \partial^{\alpha}
$$

where $a_{\alpha}$ 's are reasonably "nice" functions on $\Omega$, generates differential operators on $L^{2}\left(\mathbf{R}^{n}\right)$ and $L^{2}(\Omega, \mu)$. That is, on $L^{2}\left(\mathbf{R}^{n}\right)$, we have a family of operators

$$
\left(A_{\omega} f\right)(t)=\left.\sum_{\alpha_{1}, \ldots, \alpha_{n}} a_{\alpha_{1} \cdots \alpha_{n}}(\omega+t) \frac{\partial^{\alpha_{1}} \cdots \partial^{\alpha_{n}} f\left(t+\left(s_{1}, \ldots, s_{n}\right)\right)}{\partial s_{1}^{\alpha_{1}} \cdots \partial s_{n}^{\alpha_{n}}}\right|_{s_{1}=\cdots=s_{n}=0}
$$

parameterized by $\omega$. Similarly, on $L^{2}(\Omega)$, we have a family of operators

$$
\begin{aligned}
\left(A^{\gamma} \xi\right)(\omega)= & \sum_{\alpha_{1}, \ldots, \alpha_{n}} a_{\alpha_{1} \cdots \alpha_{n}}(\omega) \\
& \times\left.\frac{\partial^{\alpha_{1} \cdots \partial^{\alpha_{n}}} \exp \left(-i\left\langle\left(s_{1}, \ldots, s_{n}\right), \gamma\right)\right) \xi\left(\omega+\left(s_{1}, \ldots, s_{n}\right)\right)}{\partial s_{1}^{\alpha_{1}} \cdots \partial s_{n}^{\alpha_{n}}}\right|_{s_{1}=\cdots=s_{n}=0}
\end{aligned}
$$

Received by the editors July 29, 1993 and, in revised form, November 16, 1993.

1991 Mathematics Subject Classification. Primary 35S05, 47G30.

Key words and phrases. Pseudo-differential operator, spectrum, dynamical system.

Research supported in part by National Science Foundation grant DMS-9101496. 
parameterized by $\gamma \in \widehat{\mathbf{R}}^{n}$. (Throughout the paper, $\widehat{\mathbf{R}}^{n}$ denotes the dual of $\mathbf{R}^{n}$. The benefit of making a formal distinction between $\mathbf{R}^{n}$ and $\widehat{\mathbf{R}}^{n}$ in this paper will become apparent later on.) In the case where these operators are selfadjoint, one asks what is the relation between the spectra $\sigma\left(A_{\omega}\right)$ and $\sigma\left(A^{\gamma}\right)$ ? Does $\sigma\left(A^{\gamma}\right)$ coincide with $\sigma\left(A_{\omega}\right)$ ? Or, failing that, does one spectrum at least contain the other? And of course one can ask such questions in the general case where $A_{\omega}$ and $A^{\gamma}$ are pseudo-differential operators generated by the same symbol function. There are many articles in the literature which address these questions $[2,3,5,10,14,16]$. The roots of these questions can be traced back to the study of Schrödinger operators with almost periodic coefficients. But in recent years it becomes more and more apparent that the question of the coincidence of these spectra should be understood in the context of the representation theory of the group transformation $C^{*}$-algebra (also known as the crossed product $C^{*}$-algebra) associated with the flow $\left\{(\Omega, \mathscr{B}, \mu), \mathbf{R}^{n}\right\}$. In the case where $\Omega$ is a compact Hausdorff space and the flow is topologically continuous, the crossed product $C(\Omega) \times \mathbf{R}^{n}$ has natural representations $\pi_{\omega}$ on $L^{2}\left(\mathbf{R}^{n}\right)$ and $\pi^{\gamma}$ on $L^{2}(\Omega)$. For an elliptic operator of positive order, we have $\left(A_{\omega}-z\right)^{-1} \in \pi_{\omega}\left(C(\Omega) \times \mathbf{R}^{n}\right)$ and $\left(A^{\gamma}-z\right)^{-1} \in \pi^{\gamma}\left(C(\Omega) \times \mathbf{R}^{n}\right)$ [9]. Therefore the relation between $\sigma\left(A_{\omega}\right)$ and $\sigma\left(A^{\gamma}\right)$ is determined by the representations $\pi_{\omega}$ and $\pi^{\gamma}$. For example, if the flow happens to be free and minimal, then it is well known that $\sigma\left(A_{\omega}\right)=\sigma\left(A^{\gamma}\right)$. This approach to the spectra of $A_{\omega}$ and $A^{\gamma}$ is heavily influenced by similar techniques employed in the study of representations of Toeplitz $C^{*}$-algebras [4, 5, 7]. Also see [12, 13].

But in the pure measure-theoretical setting, i.e., when $(\Omega, \mathscr{B}, \mu)$ is only a measure space, the relation between $\sigma\left(A_{\omega}\right)$ and $\sigma\left(A^{\gamma}\right)$ is much less clear. However, there have been a number of articles devoted to this subject. The article that is most relevant to the present study is that of S. M. Kozlov and M. A. Shubin [10]. In that article, the authors introduced the family of operators $\left\{A_{\omega}: \omega \in \Omega\right\}$ on the space $L^{2}\left(\mathbf{R}^{n} / \Gamma\right)$, where $\Gamma$ is the lattice of periods of the dynamical system. But of the family of operators $\left\{A^{\gamma}: \gamma \in \widehat{\mathbf{R}}^{n}\right\}$, only $A^{0}$ was considered in [10]. Under the assumptions that the measure $\mu$ is ergodic and that $A_{\omega}$ 's are elliptic, selfadjoint operators of positive order, Kozlov and Shubin proved in [10] that $\sigma\left(A_{\omega}\right)=\sigma\left(A^{0}\right)$ for almost every $\omega \in \Omega$. The proof of this spectral coincidence relies on the fact that when the order of $A_{\omega}$ is positive, for each $\varphi \in C_{c}^{\infty}(\mathbf{R})$, there is a nice kernel function $K_{\varphi}(\omega, s)$ such that $\left(\varphi\left(A_{\omega}\right) f\right)(t)=\int K_{\varphi}(\omega+t, s) f(t+s) d s$ and $\left(\varphi\left(A^{0}\right) \xi\right)(\omega)=\int K_{\varphi}(\omega, s) \xi(\omega+s) d s$. The ergodicity was used to show that the time averages of both $\varphi\left(A_{\omega}\right)$ and $\varphi\left(A^{0}\right)$ have $\int_{\Omega} K_{\varphi}(\omega, s) d \mu(\omega)$ as a kernel function on their respective spaces. Therefore $\varphi\left(A^{0}\right)=0$ if and only if $\varphi\left(A_{\omega}\right)=0$ for almost every $\omega$. Here, the use of time mean and space mean of the operators is reminiscent of similar techniques used in the earlier studies of $C^{*}$-algebras of almost periodic Toeplitz operators [4, 5, 7]. Of course in Kozlov and Shubin's work, since they were dealing with measurable flows rather than almost periodic flows, the computation of these means had to be handled more carefully.

We will take one step further in the investigation of $\sigma\left(A_{\omega}\right)$ and $\sigma\left(A^{\gamma}\right)$. In this paper, we will not assume that the dynamical system $\left\{(\Omega, \mathscr{B}, \mu), \mathbf{R}^{n}\right\}$ is ergodic, nor will we assume that the orders of our pseudo-differential operators 
are positive ${ }^{1}$. The techniques employed in [10] will not help as much. This is because, first of all, if the operators $A_{\omega}$ are not assumed to have positive order, then it is not clear that one still has a kernel function for $\varphi\left(A_{\omega}\right), \varphi \in C_{c}^{\infty}(\mathbf{R})$. Secondly, even if the kernel function $K_{\varphi}$ for $\varphi\left(A_{\omega}\right)$ exists, without ergodicity, its time average is only an invariant function and is not completely independent of the $\omega$-variable. Therefore such an average has little use for the purpose of analyzing $\sigma\left(A_{\omega}\right)$ and $\sigma\left(A^{\gamma}\right)$. Our approach to the question of spectral coincidence is completely different from that of [10].

Because our dynamical systems are not assumed to be ergodic in general, we can no longer expect $\sigma\left(A_{\omega}\right)$ to be independent of $\omega$ almost everywhere. In fact one can give trivial examples where $\sigma\left(A_{\omega}\right)$ varies in $\omega$. But one can still ask what is the relation between $\sigma\left(A_{\omega}\right)$ and $\sigma\left(A^{\gamma}\right)$ ? We approach this question in the following way. We will first impose a mild assumption on the dynamical system which will eventually be dropped. That is, we first assume that there is a closed subgroup $\Gamma^{\prime} \subset \widehat{\mathbf{R}}^{n}$ such that on any invariant set $\Delta \in \mathscr{B}$ of positive measure, the joint spectrum of the $n$ partial derivatives along the orbits of the flow is $\Gamma^{\prime}$. This condition is automatically satisfied in the case $\mu$ is ergodic [10]. But in general this is a much weaker assumption than ergodicity. Then we introduce $\Gamma=\left\{t \in \mathbf{R}^{n}:\langle t, \gamma\rangle \in 2 \pi \mathbf{Z}\right.$ for every $\left.\gamma \in \Gamma^{\prime}\right\}$. We will introduce an operator $A$ on $L^{2}\left(\Omega \times\left(\mathbf{R}^{n} / \Gamma\right)\right)=L^{2}(\Omega) \otimes L^{2}\left(\mathbf{R}^{n} / \Gamma\right)$, which is unitarily equivalent to both the direct integral of $\left\{A_{\omega}: \omega \in \Omega\right\}$ and that of $\left\{A^{\gamma}: \gamma \in \Gamma^{\prime}\right\}$. In fact if $A_{\omega}$ and $A^{\gamma}$ are induced by the differential expression $(0.1)$, then

$$
\begin{aligned}
(A \xi)(\omega, t)= & \sum_{\alpha_{1}, \ldots, \alpha_{m}} a_{\alpha_{1} \cdots \alpha_{n}}(\omega) \\
& \times\left.\frac{\partial^{\alpha_{1}} \cdots \partial^{\alpha_{n}} \xi\left(\omega+\left(s_{1}, \ldots, s_{n}\right), t+\left(s_{1}, \ldots, s_{n}\right)\right)}{\partial s_{1}^{\alpha_{1}} \cdots \partial s_{n}^{\alpha_{n}}}\right|_{s_{1}=\cdots=s_{n}=0} .
\end{aligned}
$$

We will prove that if $A$ is selfadjoint, then $\sigma(A)=\sigma\left(A^{\gamma}\right)$ for every $\gamma \in \Gamma^{\prime}$. Actually it is quite easy to show that $\sigma(A) \supset \sigma\left(A^{\gamma}\right)$ for almost every $\gamma \in \Gamma^{\prime}$ with respect to the Haar measure on $\Gamma^{\prime}$ and that $\bigcup_{\gamma \in \Gamma^{\prime} \backslash N} \sigma\left(A^{\gamma}\right)=\sigma(A)$ with some null set $N \subset \Gamma^{\prime}$. If $\gamma-\gamma^{\prime}$ is an eigenvalue of the dynamical system, i.e., if there is a unimodular function $\xi$ on $\Omega$ such that $\xi(\omega+t)=\exp \left(i\left\langle t, \gamma-\gamma^{\prime}\right\rangle\right) \xi(\omega)$, then obviously $A^{\gamma}$ and $A^{\gamma^{\prime}}$ are unitarily equivalent and, therefore, $\sigma\left(A^{\gamma}\right)=$ $\sigma\left(A^{\gamma^{\prime}}\right)$. Recall the following elementary fact in operator theory: If $\left\{B_{n}\right\}$ is a sequence of selfadjoint operators whose spectra are identical and $B_{n} \rightarrow B$ in the strong operator topology, then $\sigma(B) \subset \sigma\left(B_{n}\right)$. This simple fact of operator theory tells us that if it happens that the eigenvalues of the dynamical system are dense in $\Gamma^{\prime}$, then $\sigma\left(A^{\gamma}\right)$ is independent of $\gamma$ and $\sigma(A)=\sigma\left(A^{\gamma}\right)$. But the fact of the matter is that the dynamical system may not have any eigenvalue other than 0 . Nevertheless, we are able to find an acceptable substitute for eigenvectors of the dynamical system which allows us to establish the fact that $\sigma\left(A^{\gamma}\right)$ is independent of $\gamma \in \Gamma^{\prime}$. This substitute is what one might call approximate eigenvectors of the flow. On the other hand, it is relatively easy to show that $\sigma(A) \supset \sigma\left(A_{\omega}\right)$ for a.e. $\omega \in \Omega$. Therefore we can assert that $\sigma\left(A^{\gamma}\right) \supset \sigma\left(A_{\omega}\right)$ for every $\gamma \in \Gamma^{\prime}$ and almost every $\omega \in \Omega$. This approach to the spectra of random pseudo-differential operators is motivated by recent developments in

\footnotetext{
${ }^{1}$ One of the questions left open in [10] is what happens if the order of $A_{\omega}$ is not positive.
} 
the study of Toeplitz algebras associated with topological dynamical systems where the technique of approximate eigenvectors (in the topological setting) was first introduced [13].

Our second objective is to determine $\sigma(A)$ analytically. Let $d m$ denote the Haar measure on $\Gamma^{\prime}$. For each $R>0$, let $B_{R}=\left\{\gamma \in \Gamma^{\prime}:|\gamma|<R\right\}$. There is a finite Borel measure $V_{R}$ on $\mathbf{R}$ such that

$$
\int_{B_{R}}\left\langle\left(A^{\gamma}-z\right)^{-1} 1,1\right\rangle d m(\gamma)=\int_{\mathbf{R}} \frac{1}{t-z} d V_{R}(t)
$$

for every $z \in \mathbf{C} \backslash \mathbf{R}$. It is easy to see that if $R<R^{\prime}$, then $V_{R^{\prime}}-V_{R}$ is a positive measure. We will prove that $\sigma(A)$ is the collection of $\lambda \in \mathbf{R}$ which has the property that for each $\varepsilon>0$ there exists an $R=R(\varepsilon)>0$ such that $V_{R}((\lambda-\varepsilon, \lambda+\varepsilon))>0$.

Finally, after having obtained these results under the assumption imposed on the dynamical systems earlier, we will work with arbitrary dynamical systems. The trick here is that in the case where there is no such $\Gamma^{\prime}$ associated with $\left\{(\Omega, \mathscr{B}, \mu), \mathbf{R}^{n}\right\}$, we will consider the infinite product space $\Omega^{\#}=\Omega \times \Omega \times \cdots$ with the product measure $\mu^{\#}=\mu \times \mu \times \cdots$. The flow on $\Omega$ naturally induces a flow on $\Omega^{\#}$. As it turns out, if $\Gamma$ is the lattice of periods for the original flow $\left\{(\Omega, \mathscr{B}, \mu), \mathbf{R}^{n}\right\}$, then $\Gamma^{\prime}=\left\{\gamma \in \widehat{\mathbf{R}}^{n}:\langle t, \gamma\rangle \in 2 \pi \mathbf{Z}\right.$ for every $\left.t \in \Gamma\right\}$ is the joint spectrum for the partial differentiations on every invariant set $\Delta^{\#} \subset \Omega^{\#}$ of positive measure. In other words, there is always a $\Gamma^{\prime}$ associated with the product flow on $\left(\Omega^{\#}, \mathscr{B}^{\#}, \mu^{\#}\right)$. In a natural way $(0.1)$ is a differential expression associated with the product flow $\left\{\left(\Omega^{\#}, \mathscr{B}^{\#}, \mu^{\#}\right), \mathbf{R}^{n}\right\}$. Therefore if we replace $\left\{(\Omega, \mathscr{B}, \mu), \mathbf{R}^{n}\right\}$ by the product flow and use $(0.1)$ to define operators $A^{\# \gamma}$ on $L^{2}\left(\Omega^{\#}\right)$ instead of the space $L^{2}(\Omega)$, then we still have $\sigma\left(A^{\# \gamma}\right) \supset \sigma\left(A_{\omega}\right)$ for every $\gamma \in \Gamma^{\prime}$ and almost every $\omega \in \Omega$.

The rest of the paper is organized as follows. In $\S 1$, we prove the existence of the "approximate eigenvectors" of the dynamical systems. This is the technical preparation for the study of the spectra of our pseudo-differential operators. We introduce the pseudo-differential operators and state the main theorems in $\S 2$. These theorems are stated under the assumption about the dynamical systems mentioned earlier. Section 3 contains the technical details of the proof of these theorems. In $\S 4$, we show that for a dynamical system which is completely arbitrary, the theorems stated in $\S 2$ are still valid if we replace $\Omega$ by $\Omega^{\#}=$ $\Omega \times \Omega \times \cdots$.

\section{DyNamical SYSTEMS}

Let $(\Omega, \mathscr{B}, \mu)$ be a probability space; i.e., $\mathscr{B}$ is a $\sigma$-algebra of subsets of $\Omega$ and $\mu$ is a probability measure on $\mathscr{B}$. Suppose that $(\Omega, \mathscr{B}, \mu)$ possesses a group of measure-preserving transformations $\left\{\alpha_{t}: t \in \mathbf{R}^{n}\right\}$. For each $t \in$ $\mathbf{R}^{n}, u_{t} f=f \circ \alpha_{t}$ defines a unitary operator on the Hilbert space $L^{2}(\Omega)=$ $L^{2}(\Omega, \mathscr{B}, \mu)$. We make the following two assumptions on $(\Omega, \mathscr{B}, \mu)$ and $\left\{\alpha_{t}: t \in \mathbf{R}^{n}\right\}$ which will be in force for the rest of the paper:

The Hilbert space $L^{2}(\Omega, \mathscr{B}, \mu)$ is separable .

By the second assumption, the unitary group $\left\{u_{t}: t \in \mathbf{R}^{n}\right\}$ has the spectral 
decomposition

$$
u_{t}=\int_{\widehat{\mathbf{R}}^{n}} \exp (i\langle t, \gamma\rangle) d e(\gamma), \quad t \in \mathbf{R}^{n} .
$$

Let $\mathscr{B}_{0}$ be the $\sigma$-subalgebra which consists of $\Delta \in \mathscr{B}$ such that

$$
\mu\left(\left[\Delta \backslash \alpha_{t}(\Delta)\right] \cup\left[\alpha_{t}(\Delta) \backslash \Delta\right]\right)=0
$$

for every $t \in \mathbf{R}^{n}$. Then the collection of invariant functions in $L^{2}(\Omega)$ is $L^{2}\left(\Omega, \mathscr{B}_{0}, \mu\right)$. Alternately, $L^{2}\left(\Omega, \mathscr{B}_{0}, \mu\right)=\left\{f \in L^{2}(\Omega, \mathscr{B}, \mu): u_{t} f=f\right.$ for every $\left.t \in \mathbf{R}^{n}\right\}$. The orthogonal projection $P_{0}: L^{2}(\Omega) \rightarrow L^{2}\left(\Omega, \mathscr{B}_{0}, \mu\right)$ is a conditional expectation in the sense that $P_{0} g f=g P_{0} f$ for $g \in L^{\infty}\left(\Omega, \mathscr{B}_{0}, \mu\right)$ and $f \in L^{2}(\Omega)$. By the pointwise ergodic theorem, we have

$$
\lim _{T \rightarrow \infty} \int_{B_{T}} f\left(\alpha_{t}(\omega)\right) d t /\left|B_{T}\right|=\left(P_{0} f\right)(\omega)
$$

for a.e. $\omega \in \Omega$. Here, $B_{T}=\left\{t \in \mathbf{R}^{n}:|t| \leq T\right\}$ and $\left|B_{T}\right|$ is the Lebesgue measure of $B_{T}$. Hence $P_{0}$ has the property that if $f \in L^{\infty}(\Omega, \mathscr{B}, \mu)$, then $P_{0} f \in L^{\infty}\left(\Omega, \mathscr{B}_{0}, \mu\right)$ and $\left\|P_{0} f\right\|_{\infty} \leq\|f\|_{\infty}$. On the other hand, the mean ergodic theorem tells us that $\lim _{T \rightarrow \infty} \int_{B_{T}} u_{t} d t /\left|B_{T}\right|=P_{0}$ in the strong operator topology.

Suppose that $\Delta \in \mathscr{B}_{0}$. Then the subspace $L^{2}(\Delta)=\chi_{\Delta} L^{2}(\Omega, \mathscr{B}, \mu)$ is invariant under the unitary group $\left\{u_{t}: t \in \mathbf{R}^{n}\right\}$. Therefore $L^{2}(\Delta)$ is also an invariant subspace for the spectral measure $e$ and, consequently, $e \mid L^{2}(\Delta)=\chi_{\Delta} e$ is also a spectral measure. Let $S_{\Delta}$ denote the support of the spectral measure $e \mid L^{2}(\Delta)$. (As usual, the support of a Borel measure is defined to be the smallest closed set on which the measure is concentrated.)

Theorem 1.1. Let $G \subset \widehat{\mathbf{R}}^{n}$ be an open set. Then the following are equivalent:

(i) For every $\Delta \in \mathscr{B}_{0}$ with $\mu(\Delta)>0, S_{\Delta} \cap G \neq \varnothing$.

(ii) The norm closure of $C=\left\{\left|f_{1}\right|^{2}+\cdots+\left|f_{k}\right|^{2}: k \in \mathbf{Z}_{+}, f_{1}, \ldots, f_{k} \in\right.$ $\left.e(G) L^{2}(\Omega)\right\}$ in $L^{1}(\Omega)=L^{1}(\Omega, \mathscr{B}, \mu)$ contains the constant function 1 .

Proof. (ii) $\Rightarrow$ (i). It follows from (ii) that for any invariant set $\Delta, \chi_{\Delta}$ is contained in the $L^{1}$-closure of $\left\{\left|g_{1}\right|^{2}+\cdots+\left|g_{k}\right|^{2}: k \in \mathbf{Z}_{+}, g_{1}, \ldots, g_{k} \in\right.$ $\left.\chi_{\Delta} e(G) L^{2}(\Omega)\right\}$. Hence if $\chi_{\Delta} \neq 0$ in $L^{2}(\Omega)$, then $e(G) L^{2}(\Delta)=\chi_{\Delta} e(G) L^{2}(\Omega) \neq$ $\{0\}$.

(i) $\Rightarrow$ (ii). Suppose the contrary. Then by the Hahn-Banach separation theorem, there would be a real function $h \in L^{\infty}(\Omega)=L^{\infty}(\Omega, \mathscr{B}, \mu)$ and a $\beta \in \mathbf{R}$ such that

$$
\int_{\Omega} h d \mu>\beta \geq \int_{\Omega} h \psi d \mu
$$

for every $\psi \in C$. Because $0 \in C$, we have $\beta \geq 0$. On the other hand, since $r \psi \in C$ whenever $\psi \in C$ and $r \in \mathbf{R}_{+}$, we have $\int_{\Omega} h \psi d \mu \leq 0$. Therefore we can rewrite the above inequality as

$$
\int_{\Omega} h d \mu>0 \geq \int_{\Omega} h \psi d \mu .
$$

Notice that if $f \in e(G) L^{2}(\Omega)$, then $f \circ \alpha_{t}$ also belongs to $e(G) L^{2}(\Omega)$. Hence 
$C$ is invariant under the composition with $\alpha_{t}, t \in \mathbf{R}^{n}$. Therefore

$$
\begin{aligned}
& \int_{\Omega}\left[\int_{B_{T}} h \circ \alpha_{t} d t /\left|B_{T}\right|\right] d \mu=\int_{\Omega} h d \mu>0 \\
& \quad \geq \int_{B_{T}}\left[\int_{\Omega} h\left(\psi \circ \alpha_{-t}\right) d \mu\right] d t /\left|B_{T}\right|=\int_{\Omega}\left[\int_{B_{T}} h \circ \alpha_{t} d t /\left|B_{t}\right|\right] \psi d \mu
\end{aligned}
$$

for every $\psi \in C$. Letting $T \rightarrow \infty$, we have

$$
\int_{\Omega} h_{0} d \mu>0 \geq \int_{\Omega} h_{0} \psi d \mu,
$$

where $h_{0}$ is the invariant function $P_{0} h$. Write $h_{0}=h_{+}-h_{-}$, where both $h_{+}$ and $h_{-}$are nonnegative invariant functions and $h_{+} h_{-}=0$. Since $\int_{\Omega} h_{0} d \mu>$ 0 , we have $\int_{\Omega} h_{+} d \mu>0$. Hence there is an $\varepsilon>0$ such that the measure of $\Delta=\left\{\omega \in \Omega: h_{+}(\omega) \geq \varepsilon\right\}$ is positive. Because $h_{+} \in L^{\infty}\left(\Omega, \mathscr{B}_{0}, \mu\right)$, we have $\Delta \in \mathscr{B}_{0}$. By (i), there is an $f \in e(G) L^{2}(\Delta)$ such that $\|f\|_{2}>0$. Hence

$$
\int_{\Omega} h_{0}|f|^{2} d \mu=\int_{\Omega} h_{+}|f|^{2} d \mu \geq \varepsilon\|f\|_{2}^{2}>0 .
$$

This contradiction proves (ii).

Recall that for any $f \in L^{1}(\Omega)$, the set of functions $\{\hat{g}: g$ is a rapidly decreasing $C^{\infty}$ function on $\left.\mathbf{R}^{n}, \int_{\mathbf{R}^{n}} g(t) f \circ \alpha_{t} d t=0\right\}$ generates an ideal in $C_{0}\left(\widehat{\mathbf{R}}^{n}\right)$. Here, $\hat{g}(\gamma)=\int_{\mathbf{R}^{n}} g(t) \exp (i\langle t, \gamma\rangle) d t$. The zero set of this ideal is the spectrum $\operatorname{sp}(f ; \alpha)$ of $f$ with respect to the transformation group $\alpha=$ $\left\{\alpha_{t}: t \in \mathbf{R}^{n}\right\}$. For those who are not familiar with the spectral analysis of functions associated with transformation groups, $[1,8,11]$ are good references. A function is invariant if and only if its spectrum is $\{0\}$. For any $f \in L^{2}(\Omega)$, we have

$$
f \in e(\operatorname{sp}(f ; \alpha)) L^{2}(\Omega) .
$$

For any $\varphi \in L^{\infty}(\Omega, \mathscr{B}, \mu)$ and open set $\Lambda \subset \widehat{\mathbf{R}}^{n}$,

$$
\varphi e(\Lambda) L^{2}(\Omega) \subset e(\Lambda+\operatorname{sp}(\varphi ; \alpha)) L^{2}(\Omega) .
$$

Let $\mathscr{S}\left(\mathbf{R}^{n}\right)$ denote the collection of $C^{\infty}$ functions on $\mathbf{R}^{n}$ which together with their derivatives are rapidly decreasing. For any $f \in \mathscr{S}\left(\mathbf{R}^{n}\right)$ and $\xi \in$ $L^{2}(\Omega, \mathscr{B}, \mu)$, the convolution $f * \xi$ is defined to be

$$
(f * \xi)(\omega)=\int_{\mathbf{R}^{n}} f(t)\left(u_{-t} \xi\right)(\omega) d t=\int_{\mathbf{R}^{n}} f(-t) \xi\left(\alpha_{t}(\omega)\right) d t .
$$

Here, the integral is convergent in the $L^{2}$-norm. Let $\mathscr{S}_{0}\left(\mathbf{R}^{n}\right)$ be the collection of $f \in \mathscr{S}\left(\mathbf{R}^{n}\right)$ whose Fourier transform has a compact support. Define $\mathscr{S}_{0}(\Omega)=$ $\mathscr{S}_{0}(\Omega, \mathscr{B}, \mu)=\left\{f * \varphi: \varphi \in L^{\infty}(\Omega, \mathscr{B}, \mu), f \in \mathscr{S}_{0}\left(\mathbf{R}^{n}\right)\right\}$. Notice that for $\varphi \in L^{\infty}(\Omega)$, if $\operatorname{sp}(\varphi ; \alpha)$ is a bounded set, then $\varphi \in \mathscr{S}_{0}(\Omega)$.

For $j=1, \ldots, n$, let $\varepsilon_{j} \in \mathbf{R}^{n}$ be the vector whose $j$ th component is 1 and whose other components are 0 . Let $\delta_{j}$ denote the infinitesimal generator $\lim _{s \rightarrow 0}\left(u_{s \varepsilon_{j}}-1\right) / \sqrt{-1} s$ of the one-parameter unitary group $\left\{u_{s \varepsilon_{j}}: s \in \mathbf{R}\right\}$. Alternately, $\delta_{j}=\int_{\widehat{\mathbb{R}}^{n}}\left\langle\varepsilon_{j}, \gamma\right\rangle d e(\gamma)$. Therefore the joint spectrum of $\delta_{1}, \ldots, \delta_{n}$ is the support of the spectral measure $e$. Similarly, for any $\Delta \in \mathscr{B}_{0}$, the joint spectrum of the restriction of $\delta_{1}, \ldots, \delta_{n}$ to $L^{2}(\Delta)$ is $S_{\Delta}$. 
For $\S \S 2$ and 3 , we will assume that the dynamical system $\left\{(\Omega, \mathscr{B}, \mu),\left\{\alpha_{t}\right.\right.$ : $\left.\left.t \in \mathbf{R}^{n}\right\}\right\}$ has the property that there is a closed subgroup $\Gamma^{\prime}$ of $\widehat{\mathbf{R}}^{n}$ such that $S_{\Delta}=\Gamma^{\prime}$ for every $\Delta \in \mathscr{B}_{0}$ with positive measure. It was shown in [10] that such a condition is satisfied by every dynamical system where the measure $\mu$ is ergodic. Let

$$
\Gamma=\left\{t \in \mathbf{R}^{n}:\langle t, \gamma\rangle \in 2 \pi \mathbf{Z} \text { for every } \gamma \in \Gamma^{\prime}\right\} .
$$

For each $\Delta \in \mathscr{B}_{0}$, let $\Gamma_{\Delta}$ be the collection of $t \in \mathbf{R}^{n}$ such that $u_{t}=1$ on $L^{2}(\Delta, \mu)$. For any $t \in \Gamma$, we have $u_{t}=\int_{\Gamma^{\prime}} \exp (i\langle t, \gamma\rangle) d e(\gamma)=1$. That is, $\Gamma_{\Delta} \supset \Gamma$ for every $\Delta \in \mathscr{B}_{0}$. On the other hand, if $t_{0} \in \Gamma_{\Delta}$, then

$$
\int_{\Gamma^{\prime}} \exp \left(i\left\langle t_{0}, \gamma\right\rangle\right) d\left(e(\gamma) \mid L^{2}(\Delta)\right)=u_{t_{0}} \mid L^{2}(\Delta)=1
$$

Hence for a set $\Delta \in \mathscr{B}_{0}$ of positive measure, the assumption that $S_{\Delta}=\Gamma^{\prime}$ implies $\exp \left(i\left\langle t_{0}, \gamma\right\rangle\right)=1$ on $\Gamma^{\prime}$, which means $t_{0} \in \Gamma$. Therefore $\Gamma_{\Delta}=\Gamma$ for every $\Delta \in \mathscr{B}_{0}$ with $\mu(\Delta)>0$.

Let $d m$ and $d \tau$ denote the Haar measures on the locally compact groups $\Gamma^{\prime}$ and $\mathbf{R}^{n} / \Gamma$ respectively. For each $f \in \mathscr{S}\left(\mathbf{R}^{n}\right)$ and each $u \in L^{2}\left(\mathbf{R}^{n} / \Gamma\right)=$ $L^{2}\left(\mathbf{R}^{n} / \Gamma, d \tau\right)$, we define the convolution $f * u$ to be

$$
(f * u)(t+\Gamma)=\int_{\mathbf{R}^{n}} f(s) u(t-s+\Gamma) d s, \quad t \in \mathbf{R}^{n} .
$$

Again, the integral is understood in the norm convergence in $L^{2}\left(\mathbf{R}^{n} / \Gamma\right)$. Let $\mathscr{S}_{0}\left(\mathbf{R}^{n} / \Gamma\right)=\left\{f * u: f \in \mathscr{S}_{0}\left(\mathbf{R}^{n}\right), u \in L^{2}\left(\mathbf{R}^{n} / \Gamma\right) \cap L^{\infty}\left(\mathbf{R}^{n} / \Gamma\right)\right\}$. Finally, for $f \in$ $\mathscr{S}_{0}\left(\mathbf{R}^{n}\right)$ and $\xi \in L^{2}\left(\Omega \times\left(\mathbf{R}^{n} / \Gamma\right)\right)=L^{2}\left(\Omega \times\left(\mathbf{R}^{n} / \Gamma\right), d \mu \times d \tau\right)$, the convolution $f * \xi$ is defined to be

$$
(f * \xi)(\omega, t+\Gamma)=\int_{\mathbf{R}^{n}} f(s) \xi\left(\alpha_{-s}(\omega), t-s+\Gamma\right) d s, \quad \omega \in \Omega, t \in \mathbf{R}^{n} .
$$

Let

$$
\begin{aligned}
\mathscr{S}_{0}(\boldsymbol{\Omega} & \left.\times\left(\mathbf{R}^{n} / \Gamma\right)\right) \\
& =\left\{f * \xi: f \in \mathscr{S}_{0}\left(\mathbf{R}^{n}\right), \xi \in L^{2}\left(\boldsymbol{\Omega} \times\left(\mathbf{R}^{n} / \Gamma\right)\right) \cap L^{\infty}\left(\Omega \times\left(\mathbf{R}^{n} / \Gamma\right)\right)\right\} .
\end{aligned}
$$

Notice that the spaces $\mathscr{S}_{0}(\Omega), \mathscr{S}_{0}\left(\mathbf{R}^{n} / \Gamma\right)$, and $\mathscr{S}_{0}\left(\Omega \times\left(\mathbf{R}^{n} / \Gamma\right)\right)$ are closed under addition. This is because for any $f_{1}, f_{2} \in \mathscr{S}_{0}\left(\mathbf{R}^{n}\right)$, there is an $f \in \mathscr{S}_{0}\left(\mathbf{R}^{n}\right)$ such that $f_{1}=f * f_{1}$ and $f_{2}=f * f_{2}$.

\section{RANDOM PSEUDO-DIFFERENTIAL OPERATORS}

For $j=1, \ldots, n$, let $\partial_{j}=\partial_{t_{j}}$ denote the partial derivative

$$
\left(\partial_{j} f\right)(t)=\lim _{s \rightarrow 0} \frac{1}{s}\left[f\left(t+s \varepsilon_{j}\right)-f(t)\right]
$$

for functions on $\mathbf{R}^{n}$. (Recall that $\varepsilon_{j}$ is the vector in $\mathbf{R}^{n}$ whose $j$ th component is 1 and whose other components are 0 .) If $f$ is a function on $\mathbf{R}^{n} / \Gamma$, then we define

$$
\left(\partial_{j} f\right)(t+\Gamma)=\lim _{s \rightarrow 0} \frac{1}{s}\left[f\left(t+s \varepsilon_{j}+\Gamma\right)-f(t+\Gamma)\right], \quad t \in \mathbf{R}^{n} .
$$

As usual, for a multi-index $\beta=\left(\beta_{1}, \ldots, \beta_{n}\right),|\beta|$ means $\left|\beta_{1}\right|+\cdots+\left|\beta_{n}\right|$ and $\partial_{t}^{\beta}$ stands for $\partial_{t_{1}}^{\beta_{1}} \cdots \partial_{t_{n}}^{\beta_{n}}$. 
Definition. A measurable function $a$ on $\Omega \times \widehat{\mathbf{R}}^{n}$ is in the class $S^{m}, m \in \mathbf{R}$, if there is a set $\Lambda=\Lambda(a) \in \mathscr{B}$ of measure 0 such that for any multi-indices $\nu$ and $\beta$, there is a constant $C_{\nu \beta}>0$ such that

$$
\sup _{\omega \in \Omega \backslash \Lambda}\left|\partial_{x}^{\nu} \partial_{t}^{\beta} a\left(\alpha_{t}(\omega), x\right)\right| \leq C_{\nu \beta}(1+|x|)^{m-|\nu|}, \quad t \in \mathbf{R}^{n}, x \in \widehat{\mathbf{R}}^{n} .
$$

Given a symbol function $a \in S^{m}$, we will define three sets of pseudodifferential operators on the spaces $L^{2}(\Omega)$,

$$
L^{2}\left(\Omega \times\left(\mathbf{R}^{n} / \Gamma\right)\right)=L^{2}\left(\Omega \times\left(\mathbf{R}^{n} / \Gamma\right), d \mu \times d \tau\right)
$$

and $L^{2}\left(\mathbf{R}^{n} / \Gamma\right)$ respectively.

Suppose that an $a \in S^{m}$ is given. We have a family of convolution operators

$$
\left(a_{\omega}^{\gamma} * f\right)(t)=(2 \pi)^{-n} \int_{\widehat{\mathbf{R}}^{n}}\left[\int_{\widehat{\mathbf{R}}^{n}} \exp (i\langle t-s, x\rangle) a(\omega, x-\gamma) f(s) d s\right] d x,
$$

where $\omega \in \Omega, \gamma \in \Gamma^{\prime}$, and $f \in \mathscr{S}\left(\mathbf{R}^{n}\right)$. Notice that for each pair of fixed $t \in \mathbf{R}^{n}$ and $\gamma \in \Gamma^{\prime}$, the function $\omega \mapsto\left(a_{\omega}^{\gamma} * f\right)(t)$ belongs to $L^{\infty}(\Omega)$. And for any fixed $\gamma \in \Gamma^{\prime}, t \mapsto \operatorname{ess} \sup _{\omega \in \Omega}\left|\left(a_{\omega}^{\gamma} * f\right)(t)\right|$ is a rapidly decreasing function on $\mathbf{R}^{n}$. For $f * \varphi \in \mathscr{S}_{0}(\Omega)$, where $f \in \mathscr{S}_{0}\left(\mathbf{R}^{n}\right)$ and $\varphi \in L^{\infty}(\Omega)$, define

$$
\begin{aligned}
\left(A^{\gamma}(f * \varphi)\right)(\omega)= & \int_{\mathbf{R}^{n}}\left(a_{\omega}^{\gamma} * f\right)(t) \varphi\left(\alpha_{-t}(\omega)\right) d t \\
= & \int_{\mathbf{R}^{n}}\left[( 2 \pi ) ^ { - n } \int _ { \widehat { \mathbf { R } } ^ { n } } \left[\int_{\mathbf{R}^{n}} \exp (i\langle t-s, x\rangle)\right.\right. \\
& \times a(\omega, x-\gamma) f(s) d s] d x] \varphi\left(\alpha_{-t}(\omega)\right) d t,
\end{aligned}
$$

where we consider the convergence of the integral in the norm topology of $L^{2}(\Omega)$. In order for $A^{\gamma}$ to be well defined as a linear operator from $\mathscr{S}_{0}(\Omega)$ into $L^{2}(\Omega)$, we must establish that if $f * \varphi=g * \psi$, then $A^{\gamma}(f * \varphi)=A^{\gamma}(g * \psi)$. But this is routine; it is straightforward to verify that if $h * \xi$ is another element in $\mathscr{S}_{0}(\Omega)$, then

$$
\left\langle A^{\gamma}(f * \varphi), h * \xi\right\rangle=\left\langle f * \varphi, A^{\gamma *}(h * \xi)\right\rangle=\left\langle g * \psi, A^{\gamma *}(h * \xi)\right\rangle=\left\langle A^{\gamma}(g * \psi), h * \xi\right\rangle,
$$

where

$$
\begin{aligned}
\left(A^{\gamma *}(h * \xi)\right)(\omega) & \\
=\int_{\mathbf{R}^{n}}\left[(2 \pi)^{-n} \int_{\widehat{\mathbf{R}}^{n}}[\right. & \int_{\mathbf{R}^{n}} \exp (i\langle t-s, x\rangle) \\
\times & \times \overline{a(\omega+s-t, x-\gamma)} h(s) d s] d x] \xi\left(\alpha_{-t}(\omega)\right) d t .
\end{aligned}
$$

Hence we have a linear pseudo-differential operator $A^{\gamma}$ from $\mathscr{S}_{0}(\Omega)$ into $L^{2}(\Omega)$. The above also shows that the adjoint of $A^{\gamma}$ has a domain that contains $\mathscr{S}_{0}(\Omega)$ and is, therefore, densely defined. This means that the closure of the operator $A^{\gamma}$ defined on $\mathscr{S}_{0}(\Omega)$ is also a linear operator. We will denote the closure also by $A^{\gamma}$. Naturally, we denote the adjoint of $A^{\gamma}$ by $A^{\gamma *}$. 
For $f * \xi \in \mathscr{S}_{0}\left(\Omega \times\left(\mathbf{R}^{n} / \Gamma\right)\right)$ with $f \in \mathscr{S}_{0}\left(\mathbf{R}^{n}\right)$ and $\xi \in L^{2}\left(\Omega \times\left(\mathbf{R}^{n} / \Gamma\right)\right) \cap$ $L^{\infty}\left(\boldsymbol{\Omega} \times\left(\mathbf{R}^{n} / \Gamma\right)\right)$, define

$$
\begin{aligned}
&(A(f * \xi))(\omega, y+\Gamma)=\int_{\mathbf{R}^{n}}\left(a_{\omega}^{0} * f\right)(t) \xi\left(\alpha_{-t}(\omega), y-t\right.+\Gamma) d t \\
&=\int_{\mathbf{R}^{n}}\left[(2 \pi)^{-n} \int_{\widehat{\mathbf{R}}^{n}}\left[\int_{\mathbf{R}^{n}} \exp (i\langle t-s, x\rangle) a(\omega, x) f(s) d s\right] d x\right] \\
& \times \xi\left(\alpha_{-t}(\omega), y-t+\Gamma\right) d t .
\end{aligned}
$$

As is the case for $A^{\gamma}$, the operator $A$ has an adjoint whose domain contains $\mathscr{S}_{0}\left(\Omega \times\left(\mathbf{R}^{n} / \Gamma\right)\right)$. Therefore, the closure of $A$ on $\mathscr{S}_{0}\left(\Omega \times\left(\mathbf{R}^{n} / \Gamma\right)\right)$ is also a linear operator and we denote the closure also by $A$.

On the space $L^{2}\left(\Omega \times\left(\mathbf{R}^{n} / \Gamma\right)\right)$, define the unitary operator

$$
(W \xi)(\omega, t+\Gamma)=\xi\left(\alpha_{t}(\omega), t+\Gamma\right), \quad \omega \in \Omega, t \in \mathbf{R}^{n} .
$$

This is well defined because of the fact that for a.e. $t+\Gamma$, the function $\omega \mapsto$ $\xi\left(\alpha_{t}(\omega), t+\Gamma\right)$ is independent of the choice of $t$ in its equivalence class. The unitary operator $W$ can also be defined in the following alternate way. If we regard $\xi \in L^{2}\left(\Omega \times\left(\mathbf{R}^{n} / \Gamma\right)\right) \cong L^{2}\left(\mathbf{R}^{n} / \Gamma, L^{2}(\Omega)\right)$ as an $L^{2}(\Omega)$-valued $L^{2}$ function on $\mathbf{R}^{n} / \Gamma$, then

$$
(W \xi)(t+\Gamma)=u_{t} \xi(t+\Gamma), \quad t \in \mathbf{R}^{n} .
$$

By straightforward calculation,

$$
\begin{array}{r}
(W A(f * \xi))(\omega, y+\Gamma)=(A(f * \xi))\left(\alpha_{y}(\omega), y+\Gamma\right) \\
=\int_{\mathbf{R}^{n}}\left(a_{\alpha_{y}(\omega)}^{0} * f\right)(t) \xi\left(\alpha_{y-t}(\omega), y-t+\Gamma\right) d t \\
=\int_{\mathbf{R}^{n}}\left[(2 \pi)^{-n} \int_{\widehat{\mathbf{R}}^{n}}\left[\int_{\mathbf{R}^{n}} \exp (i\langle t-s, x\rangle) a\left(\alpha_{y}(\omega), x\right) f(s) d s\right] d x\right] \\
\times \xi\left(\alpha_{y-t}(\omega), y-t+\Gamma\right) d t, \\
=\int_{\mathbf{R}^{n}}\left[(2 \pi)^{-n} \int_{\widehat{\mathbf{R}}^{n}}\left[\int_{\mathbf{R}^{n}} \exp (i\langle t-s, x\rangle) a\left(\alpha_{y}(\omega), x\right) f(s) d s\right] d x\right] \\
\times(W \xi)(\omega, y-t+\Gamma) d t .
\end{array}
$$

This brings us to the definition of a third class of pseudo-differential operators. For each $\omega \in \Omega$ which does not belong to the null set $\Lambda=\Lambda(a)$, define

$$
\begin{aligned}
& \left(A_{\omega} u\right)(y+\Gamma)=\int_{\mathbf{R}^{n}}\left(a_{\alpha_{y}(\omega)}^{0} * f\right)(t) u(y-t+\Gamma) d t \\
& =\int_{\mathbf{R}^{n}}\left[( 2 \pi ) ^ { - n } \int _ { \widehat { \mathbf { R } } ^ { n } } \left[\int_{\mathbf{R}^{n}} \exp (i\langle t-s, x\rangle)\right.\right. \\
& \left.\left.\quad \times a\left(\alpha_{y}(\omega), x\right) f(s) d s\right] d x\right] u(y-t+\Gamma) d t
\end{aligned}
$$

when $u \in \mathscr{S}_{0}\left(\mathbf{R}^{n} / \Gamma\right)$. Once more, $A_{\omega}$ is a closable operator on $L^{2}\left(\mathbf{R}^{n} / \Gamma\right)$ and we denote its closure by the same symbol. Equation (2.1) tells us that for any $\xi \in \mathscr{S}_{0}\left(\Omega \times\left(\mathbf{R}^{n} / \Gamma\right)\right)$,

$$
(W A \xi)(\omega, y)=\left(A_{\omega}(W \xi)\right)(\omega, y) .
$$


If we decompose the space $L^{2}\left(\Omega \times\left(\mathbf{R}^{n} / \Gamma\right)\right)$ as the direct integral

then

$$
L^{2}\left(\Omega \times\left(\mathbf{R}^{n} / \Gamma\right)\right)=\int_{\Omega} \bigoplus L^{2}\left(\mathbf{R}^{n} / \Gamma\right) d \mu(\omega),
$$

$$
W A=\left[\int_{\Omega} \bigoplus A_{\omega} d \mu(\omega)\right] W .
$$

This formula should be interpreted in the sense that for each $\xi \in$ $\mathscr{S}_{0}\left(\Omega \times\left(\mathbf{R}^{n} / \Gamma\right)\right)$, the function $t+\Gamma \mapsto(W \xi)(\omega, t+\Gamma)$ belongs to $\mathscr{S}_{0}\left(\mathbf{R}^{n} / \Gamma\right)$ for a.e. $\omega \in \Omega$ and for each such $\omega$, the function $t+\Gamma \mapsto(W A \xi)(\omega, t+\Gamma)$ equals $t+\Gamma \mapsto\left(A_{\omega}(W \xi)\right)(\omega, t+\Gamma)$.

The operators $A^{\gamma}$ are also related to $A$ by a direct integral. Let $\mathscr{F}$ : $L^{2}\left(\mathbf{R}^{n} / \Gamma\right) \rightarrow L^{2}\left(\Gamma^{\prime}\right)$ be the Fourier transform

$$
(\mathscr{F} u)(\gamma)=\int_{\mathbf{R}^{n} / \Gamma} \exp (i\langle s, \gamma\rangle) u(s+\Gamma) d \tau(s+\Gamma) .
$$

Let us write

$$
(F * \xi)(\omega, t+\Gamma)=\int_{\mathbf{R}^{n}} F(\omega, s) \xi\left(\alpha_{-s}(\omega), t-s+\Gamma\right) d s
$$

when $F \in \mathscr{S}_{0}\left(\Omega \times \mathbf{R}^{n}\right)$ and $\xi \in \mathscr{S}_{0}\left(\Omega \times\left(\mathbf{R}^{n} / \Gamma\right)\right)$. Then

$$
\begin{aligned}
((1 \otimes \mathscr{F})(F * \xi))(\omega, \gamma) & =\int_{\mathbf{R}^{n} / \Gamma} \exp (i\langle t, \gamma\rangle)\left[\int_{\mathbf{R}^{n}} F(\omega, s) \xi\left(\alpha_{-s}(\omega), t-s+\Gamma\right) d s\right] d \tau(t+\Gamma) \\
= & \int_{\mathbf{R}^{n}} F(\omega, s) \exp (i\langle s, \gamma\rangle) \\
& \times\left[\int_{\mathbf{R}^{n} / \Gamma} \exp (i\langle t-s, \gamma\rangle) \xi\left(\alpha_{-s}(\omega), t-s+\Gamma\right) d \tau(t+\Gamma)\right] d s \\
= & \int_{\mathbf{R}^{n}} F(\omega, s) \exp (i\langle s, \gamma\rangle)((1 \otimes \mathscr{F}) \xi)\left(\alpha_{-s}(\omega), \gamma\right) d s \\
= & {\left[F^{\gamma}(\omega, \cdot) *((1 \otimes \mathscr{F}) \xi)(\cdot, \gamma)\right](\omega), }
\end{aligned}
$$

where

$$
F^{\gamma}(\omega, s)=\exp (i\langle s, \gamma\rangle) F(\omega, s) .
$$

For $f \in \mathscr{S}_{0}\left(\mathbf{R}^{n}\right)$ and $\xi \in \mathscr{S}_{0}\left(\Omega \times\left(\mathbf{R}^{n} / \Gamma\right)\right)$, we have

$$
\begin{aligned}
((1 \otimes \mathscr{F}) A(f * \xi))(\omega, \gamma) & =\left((1 \otimes \mathscr{F})\left(F_{f} * \xi\right)\right)(\omega, \gamma) \\
& =\left[\left(a_{\omega}^{0} * f\right)^{\gamma} *((1 \otimes \mathscr{F}) \xi)(\cdot, \gamma)\right](\omega),
\end{aligned}
$$

where, by the definition of $A$,

Notice that

$$
F_{f}(\omega, t)=\left(a_{\omega}^{0} * f\right)(t) .
$$

$$
\begin{aligned}
\left(a_{\omega}^{0} * f\right)^{\gamma}(t)=\exp (i\langle t, \gamma\rangle)\left(a_{\omega}^{0} * f\right)(t) \\
\quad=(2 \pi)^{-n} \exp (i\langle t, \gamma\rangle) \int_{\widehat{\mathbf{R}}^{n}}\left[\int_{\mathbf{R}^{n}} \exp (i\langle t-s, x\rangle) a(\omega, x) f(s) d s\right] d x \\
\quad=(2 \pi)^{-n} \int_{\widehat{\mathbf{R}}^{n}}\left[\int_{\mathbf{R}^{n}} \exp (i\langle t-s, x+\gamma\rangle) a(\omega, x) \exp (i\langle s, \gamma\rangle) f(s) d s\right] d x \\
\quad=\left(a_{\omega}^{\gamma} * f^{\gamma}\right)(t) .
\end{aligned}
$$


Hence

$$
\begin{aligned}
((1 \otimes \mathscr{F}) A(f & * \xi))(\omega, \gamma)=\left[\left(a_{\omega}^{0} * f\right)^{\gamma} *((1 \otimes \mathscr{F}) \xi)(\cdot, \gamma)\right](\omega) \\
& =\left[\left(a_{\omega}^{\gamma} * f^{\gamma}\right) *((1 \otimes \mathscr{F}) \xi)(\cdot, \gamma)\right](\omega) \\
& =\left[A^{\gamma}\left(f^{\gamma} *(1 \otimes \mathscr{F}) \xi\right)(\cdot, \gamma)\right](\omega) \\
& =\left[A^{\gamma}((1 \otimes \mathscr{F})(f * \xi))(\cdot, \gamma)\right](\omega) .
\end{aligned}
$$

This implies that if we write

$$
L^{2}\left(\Omega \times \Gamma^{\prime}\right)=\int_{\Gamma^{\prime}} \bigoplus L^{2}(\Omega) d m(\gamma)
$$

then $A$ has the direct integral decomposition

$$
(1 \otimes \mathscr{F}) A=\left[\int_{\Gamma^{\prime}} \bigoplus A^{\gamma} d m(\gamma)\right](1 \otimes \mathscr{F}) .
$$

Before stating our theorems, we would like to give a more clear formula for the operators $A, A_{\omega}$, and $A^{\gamma}$ in the case where the symbol function $a$ is that of a differential operator of order $m$. Suppose that $a(\omega, x)=\sum_{|\beta| \leq m} \varphi_{\beta}(\omega) x^{\beta}$, where $\varphi_{\beta} \in C_{b}^{\infty}(\Omega)$ and $x^{\beta}=x_{1}^{\beta_{1}} \ldots x_{n}^{\beta_{n}}$ for $x=\left(x_{1}, \ldots, x_{n}\right)$ and $\beta=$ $\left(\beta_{1}, \ldots, \beta_{n}\right)$. Then

$$
\begin{gathered}
\left(A_{\omega} u\right)(y+\Gamma)=\left.\sum_{|\beta| \leq m} \varphi_{\beta}\left(\alpha_{y}(\omega)\right) \partial_{s}^{\beta} u(y+s+\Gamma)\right|_{s=0}, \\
\left(A^{\gamma} \eta\right)(\omega)=\left.\sum_{|\beta| \leq m} \varphi_{\beta}(\omega) \partial_{s}^{\beta} \exp (-i\langle s, \gamma\rangle) \eta\left(\alpha_{s}(\omega)\right)\right|_{s=0}
\end{gathered}
$$

and

$$
(A \xi)(\omega, y+\Gamma)=\left.\sum_{|\beta| \leq m} \varphi_{\beta}(\omega) \partial_{s}^{\beta} \xi\left(\alpha_{s}(\omega), y+s+\Gamma\right)\right|_{s=0}
$$

We are now ready to state our theorems. The spectrum of a linear operator $T$ will be denoted by $\sigma(T)$. Recall that $\lambda \in \mathbf{C}$ belongs to the left spectrum of $T$ if there does not exist any bounded operator $B$ such that $B(T-\lambda)=1$ on the domain of $T$. Let $\sigma_{l}(T)$ denote the left spectrum of $T$. If $T$ happens to be selfadjoint, then $\sigma(T)=\sigma_{l}(T)$.

Theorem 1. (i) For every $\gamma \in \Gamma^{\prime}, \sigma_{l}\left(A^{\gamma}\right)=\sigma_{l}(A)$.

(ii) The operator $A$ is bounded if and only if $A^{\gamma}$ is bounded for every $\gamma \in \Gamma^{\prime}$.

(iii) In the case $A$ is bounded, $\|A\|=\left\|A^{\gamma}\right\|$ for every $\gamma \in \Gamma^{\prime}$.

(iv) In the case $A$ is bounded, $\sigma\left(A^{\gamma}\right)=\sigma(A)$ for every $\gamma \in \Gamma^{\prime}$.

Remark 2.1. The crux of this theorem is the word "every". If one replaces "every" by "almost every", then the proof of this theorem becomes much easier. For instance, the "a.e." version of statement (ii) is a simple consequence of (2.3). But for our purpose, the "a.e." version of the theorem has little value for it would not pinpoint a single $\gamma$ for which the statements hold true.

Recall that the symbol function $a(\omega, x)$ is said to be formally selfadjoint if for a.e. $\omega \in \Omega$, the operator $A_{\omega}$ is symmetric. 
Theorem 2. The following are equivalent:

(i) There is a $\gamma \in \Gamma^{\prime}$ such that $A^{\gamma}$ is a symmetric operator.

(ii) For every $\gamma \in \Gamma^{\prime}, A^{\gamma}$ is a symmetric operator.

(iii) The operator $A$ is symmetric.

(iv) $A_{\omega}$ is symmetric for a.e. $\omega \in \Omega$.

Recall that the symbol function $a \in S^{m}$ is said to be elliptic if there exist $C>$ $0, R>0$, and a set $E \in \mathscr{B}$ with $\mu(E)=0$ such that $1 /|a(\omega, x)| \leq C|x|^{-m}$ whenever $|x| \geq R$ and $\omega \in \Omega \backslash E$. It is well known that if $a$ is formally selfadjoint (i.e., if any of (i)-(iv) of Theorem 2 is satisfied) and elliptic, then for almost every $\omega \in \Omega, A_{\omega}$ is a selfadjoint operator [10,15]. Hence in this case $\left(A_{\omega}-i\right)\left(A_{\omega}+i\right)^{-1}$ is unitary for almost every $\omega$. We claim that $A$ is selfadjoint. To verify this claim, it suffices to show that the deficiency indices of $A$ are both zero. For this purpose, we think of $L^{2}\left(\Omega \times\left(\mathbf{R}^{n} / \Gamma\right)\right)$ as $L^{2}\left(\Omega, L^{2}\left(\mathbf{R}^{n} / \Gamma\right)\right)$, the collection of $L^{2}\left(\mathbf{R}^{n} / \Gamma\right)$-valued $L^{2}$-functions on $\Omega$. Suppose $\xi \in L^{2}\left(\Omega, L^{2}\left(\mathbf{R}^{n} / \Gamma\right)\right)=\int_{\Omega} \bigoplus L^{2}\left(\mathbf{R}^{n} / \Gamma\right) d \mu$ is a vector orthogonal to $\eta(\omega)\left(A_{\omega}-i\right) f$ for all $\eta \in \mathscr{S}_{0}(\Omega)$ and $f \in \mathscr{S}_{0}\left(\mathbf{R}^{n} / \Gamma\right)$. Then

$$
\int_{\Omega} \eta(\omega)\left\langle\left(A_{\omega}-i\right) f, \xi(\omega)\right\rangle d \mu(\omega)=0
$$

This means $\left\langle\left(A_{\omega}-i\right) f, \xi(\omega)\right\rangle=0$ a.e. Since $\left(A_{\omega}-i\right) \mathscr{S}_{0}\left(\mathbf{R}^{n} / \Gamma\right)$ is dense in $L^{2}\left(\mathbf{R}^{n} / \Gamma\right)$ when $A_{\omega}$ is selfadjoint and since $L^{2}\left(\mathbf{R}^{n} / \Gamma\right)$ is separable, we have $\xi(\omega)=0$ for a.e. $\omega \in \Omega$. Similarly, if $\xi$ is orthogonal to $\eta(\omega)\left(A_{\omega}+i\right) f$ for all $\eta \in \mathscr{S}_{0}(\Omega)$ and $f \in \mathscr{S}_{0}\left(\mathbf{R}^{n} / \Gamma\right)$, then $\xi=0$ a.e. By (2.2), this means the deficiency indices of $A$ are both zero. It is known that for $\gamma \in \Gamma^{\prime}, A^{\gamma}$ is selfadjoint if the symbol function $a$ is elliptic and formal selfadjoint [6].

Theorem 3. Suppose that the symbol function $a$ is elliptic and formally selfadjoint. Then $\sigma\left(A^{\gamma}\right)=\sigma(A)$ for every $\gamma \in \Gamma^{\prime}$.

Proof. Since $A^{\gamma}$ and $A$ are selfadjoint operators, we have $\sigma\left(A^{\gamma}\right)=\sigma_{l}\left(A^{\gamma}\right)$ and $\sigma(A)=\sigma_{l}(A)$. By Theorem 1(i), $\sigma_{l}\left(A^{\gamma}\right)=\sigma_{l}(A)$.

Theorem 4. Suppose that the symbol function $a$ is elliptic and formally selfadjoint. Then:

(i) For a.e. $\omega \in \Omega, \sigma\left(A_{\omega}\right) \subset \sigma(A)$.

(ii) If $A$ is bounded, then so is $A_{\omega}$ and $\left\|A_{\omega}\right\| \leq\|A\|$ for a.e. $\omega \in \Omega$.

(iii) Suppose that the measure $\mu$ is ergodic. Then $\sigma\left(A_{\omega}\right)=\sigma(A)$ for a.e. $\omega \in \Omega$. And in the case $A$ is bounded, $\left\|A_{\omega}\right\|=\|A\|$ for a.e. $\omega \in \Omega$.

Proof. Suppose that $U$ is an open disc such that $\bar{U} \cap \sigma(A)=\varnothing$. Then there is a continuous function $0 \leq \varphi \leq 1$ on $\mathrm{C}$ such that $\varphi=1$ on $U$ and $\varphi=0$ on $\sigma(A)$. Hence $\varphi(A)=0$. By (2.2), there is a set $E(U) \in \mathscr{B}$ of measure zero such that $\varphi\left(A_{\omega}\right)=0$ for every $\omega \in \Omega \backslash E(U)$. This means that $\sigma\left(A_{\omega}\right) \cap U=\varnothing$ if $\omega \epsilon$ $\Omega \backslash E(U)$. Since $C \backslash \sigma(A)$ is covered by such $U$ 's and since $\mathbf{C} \backslash \sigma(A)$ is second countable, we can find a sequence of discs $\left\{U_{n}\right\}$ such that $\bigcup_{n=1}^{\infty} U_{n}=\mathbf{C} \backslash \sigma(A)$. Let $E\left(U_{n}\right)$ be the null set corresponding to $U_{n}$. If $\omega \in \Omega \backslash\left[\bigcup_{n=1}^{\infty} E\left(U_{n}\right)\right]$, then $\sigma\left(A_{\omega}\right) \cap U_{n}=\varnothing$ for every $n$, i.e., $\sigma\left(A_{\omega}\right) \cap[\mathbf{C} \backslash \sigma(A)]=\varnothing$. This proves (i). (ii) is an immediate consequence of (2.2).

To prove (iii), we first show that there is a set $E \in \mathscr{B}$ of measure zero such that for any $f \in C_{0}(\mathbf{R})$ and $u, v \in L^{2}\left(\mathbf{R}^{n} / \Gamma\right)$, the function $\omega \mapsto\left\langle f\left(A_{\omega}\right) u, v\right\rangle$ is measurable on $\Omega \backslash E$. Let $z \in \mathbf{C} \backslash \mathbf{R}$, and let $B_{z}=W(A-z)^{-1} W^{*}$. If we 
think of $L^{2}\left(\Omega \times\left(\mathbf{R}^{n} / \Gamma\right)\right)$ as the collection of $L^{2}\left(\mathbf{R}^{n} / \Gamma\right)$-valued $L^{2}$-functions on $\Omega$, then it follows from $(2.2)$ that $W(A-z) W^{*}$ commutes with the operators of multiplication by functions in $L^{\infty}(\Omega)$. It follows from the identity $B_{z} W(A-z) W^{*}=1$ that $B_{z}$ also commutes with such multiplication operators. Hence there is an $\mathscr{L}\left(L^{2}\left(\mathbf{R}^{n} / \Gamma\right)\right)$-valued strongly measurable function $B_{z}(\omega)$ on $\Omega$ such that $B_{z}=\int_{\Omega} \oplus B_{z}(\omega) d \mu(\omega)$. Since

$$
\int_{\Omega} \bigoplus B_{z}(\omega)\left(A_{\omega}-z\right) d \mu(\omega)=B_{z} W(A-z) W^{*}=1,
$$

there is a set $E_{z} \in \mathscr{B}$ of measure zero such that $B_{z}(\omega)=\left(A_{\omega}-z\right)^{-1}$ for every $\omega \in \Omega \backslash E_{z}$. Let $\left\{z_{1}, \ldots, z_{n}, \ldots\right\}$ be a countable dense subset of $\mathbf{C} \backslash \mathbf{R}$, and let $E=\bigcup_{n=1}^{\infty} E_{z_{n}}$. Then for $n=1,2,3, \ldots$, the function $\omega \mapsto\left(A_{\omega}-z_{n}\right)^{-1}$ is strongly measurable on $\Omega \backslash E$. On the other hand, since the linear combinations of $\left\{\left(t-z_{n}\right)^{-1}\right\}_{n=1}^{\infty}$ are dense in $C_{0}(\mathbf{R})$, for every $f \in C_{0}(\mathbf{R})$, the function $\omega \mapsto f\left(A_{\omega}\right)$ is strongly measurable on $\Omega \backslash E$.

Let $\mathscr{P}$ be the collection of pairs of open intervals $\left\{(a, b),\left(a^{\prime}, b^{\prime}\right)\right\}$ where the end points $a, b, a^{\prime}$, and $b^{\prime}$ are rational numbers satisfying the relation $a<$ $a^{\prime}<b^{\prime}<b$. For each pair $P=\left\{(a, b),\left(a^{\prime}, b^{\prime}\right)\right\} \in \mathscr{P}$, choose a continuous function $0 \leq \varphi_{P} \leq 1$ on $\mathbf{R}$ such that $\varphi_{P}=1$ on $\left[a^{\prime}, b^{\prime}\right]$ and $\varphi_{P}=0$ on $\mathbf{R} \backslash(a, b)$. For any $P \in \mathscr{P}$, if $\varphi_{P}(A) \neq 0$, then the set $G_{P}=\{\omega \in \Omega \backslash E$ : $\left.\varphi_{P}\left(A_{\omega}\right) \neq 0\right\}$ is measurable and has positive measure. For each $s \in \mathbf{R}^{n}$, let $U_{s}$ be the unitary operator $\left(U_{s} u\right)(\hat{\imath}+\Gamma)=u(t+s+\Gamma)$ on $L^{2}\left(\mathbf{R}^{n} / \Gamma\right)$. It follows from the definition of $A_{\omega}$ that $U_{s} A_{\omega} U_{-s}=A_{\alpha_{s}}(\omega)$. If $f \in C_{0}(\mathbf{R})$, then $f\left(A_{\alpha_{s}(\omega)}\right)=f\left(U_{s} A_{\omega} U_{-s}\right)=U_{s} f\left(A_{\omega}\right) U_{-s}$. Hence $\alpha_{s}\left(G_{P}\right) \subset G_{P} \cup E$, where $E$ is the null set introduced in the previous paragraph. Since the measure $\mu$ is now assumed to be ergodic and $\mu\left(G_{P}\right)>0$, we have $\mu\left(G_{P}\right)=1$. Let $G$ be the intersection of all $G_{P}$ 's where $P \in \mathscr{P}$ and $\varphi_{P}(A) \neq 0$. Since $\mathscr{P}$ is a countable set, $G$ is measurable and $\mu(G)=1$. Suppose $\lambda \in \sigma(A)$. We can find a sequence $P_{n}=\left\{\left(a_{n}, b_{n}\right),\left(a_{n}^{\prime}, b_{n}^{\prime}\right)\right\}, n=1,2, \ldots$, in $\mathscr{P}$ such that $\lambda$ is contained in every $\left(a_{n}^{\prime}, b_{n}^{\prime}\right)$ and $b_{n}-a_{n} \rightarrow 0$. Since $\lambda \in \sigma(A)$ and $\varphi_{P_{n}}=1$ on a neighborhood of $\lambda, \varphi_{P_{n}}(A) \neq 0$ for every $n$. This implies that if $\omega \in G$, then $\varphi_{P_{n}}\left(A_{\omega}\right) \neq 0$ for every $n$. Fix an $\omega_{0} \in G$. Since $\varphi_{P_{n}}=0$ on $\mathbf{R} \backslash\left(a_{n}, b_{n}\right)$, we have $\sigma\left(A_{\omega_{0}}\right) \cap\left(a_{n}, b_{n}\right) \neq \varnothing$. Because $\sigma\left(A_{\omega_{0}}\right)$ is a closed set, we have $\lambda \in \sigma\left(A_{\omega_{0}}\right)$. That is, $\sigma\left(A_{\omega}\right) \supset \sigma(A)$ if $\omega \in G$.

Remark 2.2. Unlike Theorem 1, this theorem is obviously not true if one replaces "almost every" in the statement by "every".

Remark 2.3. That $\sigma\left(A_{\omega}\right)$ is independent of $\omega$ for a.e. $\omega \in \Omega$ was proved in [10, Corollary 1 in $\S 3]$ under the assumptions that the dynamical system is ergodic, that the symbol $a \in S^{m}$ is elliptic and formally selfadjoint, and that the order $m$ of $A_{\omega}$ is strictly positive. As commented in [10], the results of [10] also yield the independence of $\sigma\left(A_{\omega}\right)$ in the case $m<0$ if $a$ is invertible. However the techniques employed in [10] cannot be used to treat the case $m=0$ and the case $m<0$ if $a$ is not invertible. By contrast, Theorem 4(iii) states that the independence of $\sigma\left(A_{\omega}\right)$ is valid as long as the dynamical system is ergodic and $A$ is selfadjoint. As far as our proof is concerned, the order $m$ of the operator and the invertibility of the symbol $a$ are irrelevant. The main reason for this improvement is that we do not rely on the kernel function of $\varphi\left(A_{\omega}\right)\left(\varphi \in \mathscr{S}_{0}(\mathbf{R})\right)$, which was the main technical tool in [10]. 
Remark 2.4. The main result of [10] is that if $\mu$ is ergodic, $a$ is formally selfadjoint and elliptic, and $m>0$, then $\sigma\left(A^{0}\right)=\sigma\left(A_{\omega}\right)$ for a.e. $\omega \in \Omega$. By comparison, our results are much broader. The operators $A$ and $A^{\gamma}$ with $\gamma \neq 0$ were not even introduced in [10]. If $\mu$ is not ergodic, then obviously it is not true in general that $\sigma\left(A^{0}\right)=\sigma\left(A_{\omega}\right)$ for a.e. $\omega \in \Omega$. The right theorem is that $\sigma\left(A^{\gamma}\right) \supset \sigma\left(A_{\omega}\right)$ for every $\gamma$ and almost every $\omega \in \Omega$.

Our next theorem gives a characterization of the spectrum $\sigma(A)$ in the case $A$ is selfadjoint. For $R>0$, let $B_{R}=\left\{\gamma \in \Gamma^{\prime}:|\gamma|<R\right\}$. Consider the analytic function

$$
F_{R}(z)=\int_{B_{R}}\left\langle\left(A^{\gamma}-z\right)^{-1} 1,1\right\rangle d m(\gamma)
$$

on $\mathbf{C} \backslash \mathbf{R}$. (Here, $\left(A^{\gamma}-z\right)^{-1} 1$ is the image of the constant function 1 under the operator $\left(A^{\gamma}-z\right)^{-1}$ and $\left\langle\left(A^{\gamma}-z\right)^{-1} 1,1\right\rangle$ the $L^{2}(\Omega)$-inner product of $\left(A^{\gamma}-z\right)^{-1} 1$ with the constant function 1.) There is a positive Borel measure $V_{R}$ on $\mathrm{R}$ such that

$$
F_{R}(z)=\int_{\mathbf{R}} \frac{1}{\lambda-z} d V_{R}(\lambda) .
$$

The existence of such a measure $V_{R}$ can be proved using function theory and the properties of $F_{R}$. On the other hand, $V_{R}$ can also be obtained explicitly. Let

$$
g_{R}(\omega, t+\Gamma)=g_{R}(t+\Gamma)=\int_{\Gamma^{\prime}} \exp (-i\langle t, \gamma\rangle) \chi_{[0, R)}(|\gamma|) d m(\gamma),
$$

which is a function in $L^{2}\left(\Omega \times\left(\mathbf{R}^{n} / \Gamma\right)\right)$. It follows from (2.3) that

$$
F_{R}(z)=\left\langle(A-z)^{-1} g_{R}, g_{R}\right\rangle \text {. }
$$

Therefore if $A=\int_{\mathbf{R}} \lambda d \widetilde{E}_{\lambda}$ is the spectral decomposition of $A$, then

$$
V_{R}(\Delta)=\left\langle\widetilde{E}(\Delta) g_{R}, g_{R}\right\rangle
$$

for every Borel set $\Delta \subset \mathbf{R}$. For $R^{\prime}>R$, we have $\operatorname{Im} F_{R^{\prime}}(z) \geq \operatorname{Im} F_{R}(z)$ if $\operatorname{Im} z>0$. Hence for any Borel set $\Delta \subset \mathbf{R}$, we have $V_{R^{\prime}}(\Delta) \geq V_{R}(\Delta)$. Our next theorem asserts that $\sigma(A)$ is completely determined by the action of $\left\{\left(A^{\gamma}-z\right)^{-1}: \gamma \in \Gamma^{\prime}, z \in \mathbf{C} \backslash \mathbf{R}\right\}$ on the single vector $1 \in L^{2}(\Omega)$.

Theorem 5. Suppose that $A$ is selfadjoint. Then $\sigma(A)$ is the collection of $\lambda \in \mathbf{R}$ which satisfies the condition that for every $\varepsilon>0$, there is an $R=R(\varepsilon)>0$ such that $V_{R}((\lambda-\varepsilon, \lambda+\varepsilon))>0$.

\section{THE PROOFS OF THEOREMS 1,2 , AND 5}

By Theorem 1.1, the $L^{1}$-norm closure of $\left\{\left|f_{1}\right|^{2}+\cdots+\left|f_{k}\right|^{2}: k \in \mathbf{Z}_{+}, f_{1}, \ldots\right.$, $\left.f_{k} \in e(G) L^{2}(\Omega)\right\}$ contains the constant function 1 if $G$ is open and $G \cap \Gamma^{\prime} \neq \varnothing$. Consequently if $H$ is a dense subset of $L^{2}(\Omega)$, then the $L^{1}$-norm closure of $\left\{\left|f_{1}\right|^{2}+\cdots+\left|f_{k}\right|^{2}: k \in \mathbf{Z}_{+}, f_{1}, \ldots, f_{k} \in e(G) H\right\}$ also contains the constant function 1. We claim that for every $\gamma \in \Gamma^{\prime}$ and every positive integer $k$, there are functions $f_{k, 1}^{\gamma}, \ldots, f_{k, p_{k}}^{\gamma} \in\left[e\left(-\gamma+B_{1 / k}\right) \mathscr{S}_{0}(\Omega)\right] \cap \mathscr{S}_{0}(\Omega)$ (recall that $\left.B_{r}=\left\{\gamma \in \Gamma^{\prime}:|\gamma|<r\right\}\right)$ such that

$$
\left\|1-\left|f_{k, 1}^{\gamma}\right|^{2}-\cdots-\left|f_{k, p_{k}}^{\gamma}\right|^{2}\right\|_{1} \leq 1 / k .
$$


This is simply because

$$
\left[e\left(-\gamma+B_{1 / k}\right) \mathscr{S}_{0}(\Omega)\right] \cap \mathscr{S}_{0}(\Omega) \supset \int_{\Gamma^{\prime}} g(\lambda) d e(\lambda) \mathscr{S}_{0}(\Omega) \supset e\left(-\gamma+B_{1 / 2 k}\right) \mathscr{S}_{0}(\Omega),
$$

where $0 \leq g \leq 1$ is a $C^{\infty}$-function on $\widehat{\mathbf{R}}^{n}$ such that $g=0$ on $\widehat{\mathbf{R}}^{n} \backslash\left(B_{2 / 3 k}-\gamma\right)$ and $g=1$ on $B_{1 / 2 k}-\gamma$. It is easy to see that for any $\gamma \in \Gamma^{\prime}$ and $\eta \in L^{\infty}(\Omega)$,

$$
\lim _{k \rightarrow \infty} \sum_{j=1}^{p_{k}}\left\|f_{k, j}^{\gamma} \eta\right\|_{2}^{2}=\lim _{k \rightarrow \infty} \int_{\Omega}\left(\sum_{j=1}^{p_{k}}\left|f_{k, j}(\omega)\right|^{2}\right)|\eta(\omega)|^{2} d \mu(\omega)=\|\eta\|_{2}^{2} .
$$

Let $L$ be a Banach generalized limit on $l^{\infty}\left(\mathbf{Z}_{+}\right)$. Suppose that $T$ is a bounded operator on $L^{2}(\Omega)$ and $\eta, \psi \in L^{\infty}(\Omega)$. Then it follows from the above equality and the well-known properties of $L$ that

$$
\left|L\left(\left\{\sum_{j=1}^{p_{k}}\left\langle T f_{k, j}^{\gamma} \eta, f_{k, j}^{\gamma} \psi\right\rangle\right\}_{k=1}^{\infty}\right)\right| \leq\|T\|\|\eta\|_{2}\|\psi\|_{2} \text {. }
$$

Therefore there is a bounded operator $\Phi^{\gamma}(T)$ on $L^{2}(\Omega)$ such that

$$
\left\langle\Phi^{\gamma}(T) \eta, \psi\right\rangle=L\left(\left\{\sum_{j=1}^{p_{k}}\left\langle T f_{k, j}^{\gamma} \eta, f_{k, j}^{\gamma} \psi\right\rangle\right\}_{k=1}^{\infty}\right)
$$

It is obvious that the map $T \mapsto \Phi^{\gamma}(T)$ is a linear transformation on $\mathscr{L}\left(L^{2}(\Omega)\right)$ whose norm equals 1 . In fact the argument in the previous paragraph shows that $\Phi^{\gamma}(1)=1$, an important fact to bear in mind. It is also obvious that $\Phi^{\gamma}$ maps selfadjoint operators to selfadjoint operators and nonnegative operators to nonnegative operators.

We will next define and compute $\Phi^{\gamma}\left(A^{\nu}\right)$. Suppose that $\eta \in \mathscr{S}_{0}(\Omega)$. Then there is a bounded open set $G$ such that $\eta \in e(G) L^{2}(\Omega)$. By (1.4), $f_{k, j}^{\gamma} \eta \in$ $e\left(-\gamma+B_{1 / k}+G\right) L^{2}(\Omega)$. Hence there is a $C^{\infty}$-function $f$ whose Fourier transform has a compact support on $\widehat{\mathbf{R}}^{n}$ such that $f_{k, j}^{\gamma} \eta=f *\left(f_{k, j}^{\gamma} \eta\right)$ for all possible $k$ and $j$. Hence

$$
\begin{aligned}
\left(A^{\nu} f_{k, j}^{\gamma} \eta\right)(\omega) & =\left(A^{\nu} f *\left(f_{k, j}^{\gamma} \eta\right)\right)(\omega) \\
& =\int_{\mathbf{R}^{n}}\left(a_{\omega}^{\nu} * f\right)(t)\left(f_{k, j}^{\gamma} \eta\right)\left(\alpha_{-t}(\omega)\right) d t
\end{aligned}
$$

where

$$
\left(a_{\omega}^{\nu} * f\right)(t)=(2 \pi)^{-n} \int_{\widehat{\mathbf{R}}^{n}}\left[\int_{\mathbf{R}^{n}} \exp (i\langle t-s, x\rangle) a(\omega, x-\nu) f(s) d s\right] d x .
$$

The map $\varphi(\omega) \mapsto \int_{\mathbf{R}^{n}}\left(a_{\omega^{\nu}}^{\nu} f\right)(t)(\varphi)\left(\alpha_{-t}(\omega)\right) d t$ is a bounded operator on $L^{2}(\Omega)$. Hence there is a constant $C(\eta)>0$ such that $\left\|A^{\nu} f_{k, j}^{\gamma} \eta\right\|_{2} \leq C(\eta)\left\|f_{k, j}^{\gamma} \eta\right\|_{2}$. Therefore

$$
\left|L\left(\left\{\sum_{j=1}^{p_{k}}\left\langle A^{\nu} f_{k, j}^{\gamma} \eta, f_{k, j}^{\gamma} \psi\right\rangle\right\}_{k=1}^{\infty}\right)\right| \leq C(\eta)\|\eta\|_{2}\|\psi\|_{2}
$$


for every $\psi \in L^{\infty}(\Omega)$. Hence there is an element which we denote by $\Phi^{\gamma}\left(A^{\nu}\right) \eta$ in $L^{2}(\Omega)$ such that

$$
\left\langle\Phi^{\gamma}\left(A^{\nu}\right) \eta, \psi\right\rangle=L\left(\left\{\sum_{j=1}^{p_{k}}\left\langle A^{\nu} f_{k, j}^{\gamma} \eta, f_{k, j}^{\gamma} \psi\right\rangle\right\}_{k=1}^{\infty}\right)
$$

Thus we have a linear transformation $\Phi^{\gamma}\left(A^{\nu}\right)$ from $\mathscr{S}_{0}(\Omega)$ into $L^{2}(\Omega)$. For a bounded linear operator $T$ on $L^{2}(\Omega)$, we similarly have a linear transformation $\Phi^{\gamma}\left(T A^{\nu}\right)$ from $\mathscr{S}_{0}(\Omega)$ into $L^{2}(\Omega)$ such that

$$
\left\langle\Phi^{\gamma}\left(T A^{\nu}\right) \eta, \psi\right\rangle=L\left(\left\{\sum_{j=1}^{p_{k}}\left\langle T A^{\nu} f_{k, j}^{\gamma} \eta, f_{k, j}^{\gamma} \psi\right\rangle\right\}_{k=1}^{\infty}\right)
$$

holds for all $\eta \in \mathscr{S}_{0}(\Omega)$ and $\psi \in L^{\infty}(\Omega)$. Following the same line of reasoning, we can also define $\Phi^{\gamma}\left(A^{\nu *}\right)$ and $\Phi^{\gamma}\left(T A^{\nu *}\right)$. It is clear that $\mathscr{S}_{0}(\Omega)$ is contained in the domains of $\Phi^{\gamma}\left(A^{\nu}\right)^{*}$ and $\Phi^{\gamma}\left(A^{\nu *}\right)$. Therefore both operators are closable. We will use the same symbol to denote the closure of $\Phi^{\gamma}\left(A^{\nu}\right)$. On the other hand, we treat $\Phi^{\gamma}\left(A^{\nu *}\right)$ only as an operator defined on $\mathscr{S}_{0}(\Omega)$. This is because in general we do not know whether the closure of $\Phi^{\gamma}\left(A^{\nu *}\right)$ coincides with the adjoint of $\Phi^{\gamma}\left(A^{\nu}\right)$.

Lemma 3.1. (i) Let $b(\omega, t)$ be a measurable function on $\Omega \times \mathbf{R}^{n}$ such that the function $t \mapsto\|b(\cdot, t)\|_{\infty}$ belongs to $L^{1}\left(\mathbf{R}^{n}\right)$. Let $T$ be the (bounded) operator on $L^{2}(\Omega)$ such that $(T \varphi)(\omega)=\int_{\mathbf{R}^{n}} b(\omega, t) \varphi\left(\alpha_{-t}(\omega)\right) d t$. Then

$$
\Phi^{\gamma}(T)=T^{\gamma}
$$

where

$$
\left(T^{\gamma} \varphi\right)(\omega)=\int_{\mathbf{R}^{n}} b(\omega, t) \exp (i\langle t, \gamma\rangle) \varphi\left(\alpha_{-t}(\omega)\right) d t
$$

for $\varphi \in L^{2}(\Omega)$.

(ii) Let $T$ and $T^{\gamma}$ be as above. Let $B$ be a bounded operator on $L^{2}(\Omega)$. Then

$$
\boldsymbol{\Phi}^{\gamma}(B T)=\Phi^{\gamma}(B) T^{\gamma}
$$

Proof. (i) Let $\psi \in L^{\infty}(\Omega)$. Then

$$
\begin{aligned}
\left(T f_{k, j}^{\gamma} \psi\right)(\omega)= & \int_{\mathbf{R}^{n}} b(\omega, t) f_{k, j}^{\gamma}\left(\alpha_{-t}(\omega)\right) \psi\left(\alpha_{-t}(\omega)\right) d t \\
= & \int_{\mathbf{R}^{n}} b(\omega, t)\left[f_{k, j}^{\gamma}\left(\alpha_{-t}(\omega)\right)-\exp (i\langle t, \gamma\rangle) f_{k, j}^{\gamma}(\omega)\right] \psi\left(\alpha_{-t}(\omega)\right) d t \\
& +f_{k, j}^{\gamma}(\omega)\left(T^{\gamma} \psi\right)(\omega)
\end{aligned}
$$


Let $C=\int_{\mathbf{R}^{n}}\|b(\cdot, t)\|_{\infty} d t$. We have

$$
\begin{aligned}
\int_{\Omega} \mid & \left.\int_{\mathbf{R}^{n}} b(\omega, t)\left[f_{k, j}^{\gamma}\left(\alpha_{-t}(\omega)\right)-\exp (i\langle t, \gamma\rangle) f_{k, j}^{\gamma}(\omega)\right] \psi\left(\alpha_{-t}(\omega)\right) d t\right|^{2} d \mu(\omega) \\
\leq & \int_{\Omega} \int_{\mathbf{R}^{n}}|b(\omega, t)|\left|\left[f_{k, j}^{\gamma}\left(\alpha_{-t}(\omega)\right)-\exp (i\langle t, \gamma\rangle) f_{k, j}^{\gamma}(\omega)\right]\right|^{2} d t \\
& \times \int_{\mathbf{R}^{n}}|b(\omega, t)|\left|\psi\left(\alpha_{-t}(\omega)\right)\right|^{2} d t d \mu(\omega) \\
\leq & C\left\|\psi^{2}\right\|_{\infty} \int_{\mathbf{R}^{n}}\|b(\cdot, t)\|_{\infty}\left\|u_{-t} f_{k, j}^{\gamma}-\exp (i\langle t, \gamma\rangle) f_{k, j}^{\gamma}\right\|_{2}^{2} d t
\end{aligned}
$$

Let

$$
C_{k}(t)=\max \{|1-\exp (i\langle t, \lambda\rangle)|:|\lambda| \leq 1 / k\} .
$$

By our choice, $f_{k, j}^{\gamma} \in e\left(-\gamma+B_{1 / k}\right) L^{2}(\Omega)$. Hence

$$
\begin{aligned}
& \left\|u_{-t} f_{k, j}^{\gamma}-\exp (i\langle t, \gamma\rangle) f_{k, j}^{\gamma}\right\|_{2} \\
& \quad=\left\|\int_{-\gamma+B_{1 / k}}[\exp (-i\langle t, \lambda\rangle)-\exp (i\langle t, \gamma\rangle)] d e(\lambda) f_{k, j}^{\gamma}\right\|_{2} \leq C_{k}(t)\left\|f_{k, j}^{\gamma}\right\|_{2} .
\end{aligned}
$$

It follows from (3.1)-(3.3) that

$$
\left\|T f_{k, j}^{\gamma} \psi-f_{k, j}^{\gamma} T^{\gamma} \psi\right\|_{2}^{2} \leq C\left\|\psi^{2}\right\|_{\infty} \int_{\mathbf{R}^{n}}\|b(\cdot, t)\|_{\infty}\left(C_{k}(t)\right)^{2} d t\left\|f_{k, j}^{\gamma}\right\|_{2}^{2}
$$

Because $C_{k}(t) \leq 2$ and $\lim _{k \rightarrow \infty} C_{k}(t)=0$ for every $t \in \mathbf{R}^{n}$, we have

$$
\lim _{k \rightarrow \infty} \int_{\mathbf{R}^{n}}\|b(\cdot, t)\|_{\infty}\left(C_{k}(t)\right)^{2} d t=0
$$

Hence

$$
\begin{aligned}
\lim _{k \rightarrow \infty} & \sum_{j=1}^{p_{k}}\left\|T f_{k, j}^{\gamma} \psi-f_{k, j}^{\gamma} T^{\gamma} \psi\right\|_{2}^{2} \\
& \leq C\left\|\psi^{2}\right\|_{\infty} \lim _{k \rightarrow \infty} \int_{\mathbf{R}^{n}}\|b(\cdot, t)\|_{\infty}\left(C_{k}(t)\right)^{2} d t \sum_{j=1}^{p_{k}}\left\|f_{k, j}^{\gamma}\right\|_{2}^{2}=0 .
\end{aligned}
$$

Therefore for any $\varphi \in L^{\infty}(\Omega)$,

$$
\begin{aligned}
\lim _{k \rightarrow \infty} \mid & \left\langle T^{\gamma} \psi, \varphi\right\rangle-\sum_{j=1}^{p_{k}}\left\langle T f_{k, j}^{\gamma} \psi, f_{k, j}^{\gamma} \varphi\right\rangle \mid \\
& =\lim _{k \rightarrow \infty}\left|\sum_{j=1}^{p_{k}}\left(\left\langle f_{k, j}^{\gamma} T^{\gamma} \psi, f_{k, j}^{\gamma} \varphi\right\rangle-\left\langle T f_{k, j}^{\gamma} \psi, f_{k, j}^{\gamma} \varphi\right\rangle\right)\right| \\
& \leq \lim _{k \rightarrow \infty} \sum_{j=1}^{p_{k}}\left\|T f_{k, j}^{\gamma} \psi-f_{k, j}^{\gamma} T^{\gamma} \psi\right\|_{2}\left\|f_{k, j}^{\gamma} \varphi\right\|_{2} \\
& \leq \lim _{k \rightarrow \infty}\left(\sum_{j=1}^{p_{k}}\left\|T f_{k, j}^{\gamma} \psi-f_{k, j}^{\gamma} T^{\gamma} \psi\right\|_{2}^{2}\right)^{1 / 2}\left(\sum_{j=k}^{p_{k}}\left\|f_{k, j}^{\gamma} \varphi\right\|_{2}^{2}\right)^{1 / 2}=0 .
\end{aligned}
$$

This means $\Phi^{\gamma}(T)=T^{\gamma}$. 
(ii) Let $\psi$ and $\varphi$ be as above. Then

$$
\begin{aligned}
\lim _{k \rightarrow \infty}\left|\sum_{j=1}^{p_{k}}\left\langle B f_{k, j}^{\gamma} T^{\gamma} \psi, f_{k, j}^{\gamma} \varphi\right\rangle-\sum_{j=1}^{p_{k}}\left\langle B T f_{k, j}^{\gamma} \psi, f_{k, j}^{\gamma} \varphi\right\rangle\right| \\
\quad=\lim _{k \rightarrow \infty}\left|\sum_{j=1}^{p_{k}}\left(\left\langle B\left(f_{k, j}^{\gamma} T^{\gamma} \psi-T f_{k, j}^{\gamma} \psi\right), f_{k, j}^{\gamma} \varphi\right\rangle\right)\right| \\
\quad \leq\|B\| \lim _{k \rightarrow \infty} \sum_{j=1}^{p_{k}}\left\|T f_{k, j}^{\gamma} \psi-f_{k, j}^{\gamma} T^{\gamma} \psi\right\|_{2}\left\|f_{k, j}^{\gamma} \varphi\right\|_{2}=0 .
\end{aligned}
$$

Lemma 3.2. (i) For any $\gamma, \nu \in \Gamma^{\prime}$ and $\eta \in \mathscr{S}_{0}(\Omega)$,

$$
\Phi^{\gamma}\left(A^{\nu}\right) \eta=A^{\nu+\gamma} \eta \text {. }
$$

(ii) If $B$ is a bounded operator on $L^{2}(\Omega)$, then for any $\eta \in \mathscr{S}_{0}(\Omega)$,

$$
\Phi^{\gamma}\left(B A^{\nu}\right) \eta=\Phi^{\gamma}(B) A^{\nu+\gamma} \eta \text {. }
$$

Remark. This lemma implies that $\Phi^{\gamma}\left(A^{\nu}\right)=A^{\nu+\gamma}$. But it does not necessarily mean that the closure of $\Phi^{\gamma}\left(A^{\nu *}\right) \mid \mathscr{S}_{0}(\Omega)$ is $\left(A^{\nu+\gamma}\right)^{*}$. The fact is that $\mathscr{S}_{0}(\Omega)$ may not be a core for $\left(A^{\nu+\gamma}\right)^{*}$ when the operator $A$ fails to be elliptic.

Proof of Lemma 3.2. (i) Let $\eta \in \mathscr{S}_{0}(\Omega)$ be a function which belongs to $e\left(B_{R}\right) L^{2}(\Omega)$ with some $R>0$. Let $S=1+|\gamma|+R$. Let $0 \leq g \leq 1$ be a $C^{\infty}$-function on $\widehat{\mathbf{R}}^{n}$ such that $g=1$ on $B_{S}$ and $g=0$ on $\widehat{\mathbf{R}}^{n} \backslash B_{S+1}$. It follows from (1.4) that $f_{k, j}^{\gamma} \eta \in e\left(-\gamma+B_{1 / k}+B_{R}\right) L^{2}(\Omega)$. Therefore

$$
f_{k, j}^{y} \eta=\int_{\Gamma^{\prime}} g(\lambda) d e(\lambda) f_{k, j}^{y} \eta=\int_{\mathbf{R}^{n}} \hat{g}(t) u_{-t} d t f_{k, j}^{y} \eta,
$$

where $g(\lambda)=\int_{\mathbf{R}^{n}} \hat{g}(t) \exp (-i\langle t, \lambda\rangle) d t$. By the definition of $A^{\nu}$, we have

$$
A^{\nu} f_{k, j}^{\gamma} \eta=T f_{k, j}^{\nu} \eta
$$

where

$$
(T \varphi)(\omega)=\int_{\mathbf{R}^{n}} b(\omega, t) \varphi\left(\alpha_{-t}(\omega)\right) d t
$$

with

$$
b(\omega, t)=(2 \pi)^{-n} \int_{\widehat{\mathbf{R}}^{n}}\left[\int_{\mathbf{R}^{n}} \exp (i\langle t-s, x\rangle) a(\omega, x-\nu) \hat{g}(s) d s\right] d x .
$$

That $a \in S^{m}$ ensures that $\int_{\mathrm{R}^{n}}\|b(\cdot, t)\|_{\infty} d t<\infty$. Applying Lemma 3.1(i), we obtain that for any $\varphi \in L^{\infty}(\Omega)$,

$$
\begin{aligned}
\left\langle\Phi^{\gamma}\left(A^{\nu}\right) \eta, \varphi\right\rangle & =L\left(\left\{\sum_{j=1}^{p_{k}}\left\langle A^{\nu} f_{k, j}^{y} \eta, f_{k, j}^{y} \varphi\right\rangle\right\}_{k=1}^{\infty}\right) \\
& =L\left(\left\{\sum_{j=1}^{p_{k}}\left\langle T f_{k, j}^{y} \eta, f_{k, j}^{y} \varphi\right\rangle\right\}_{k=1}^{\infty}\right)=\left\langle\Phi^{\gamma}(T) \eta, \varphi\right\rangle .
\end{aligned}
$$


By Lemma 3.1(i), we have

$$
\begin{aligned}
&\left(\Phi^{\gamma}(T) \eta\right)(\omega)=\int_{\mathbf{R}^{n}} b(\omega, t) \exp (i\langle t, \gamma\rangle) \eta\left(\alpha_{-t}(\omega)\right) d t \\
&=\int_{\mathbf{R}^{n}}\left[(2 \pi)^{-n} \int_{\widehat{\mathbf{R}}^{n}}\left[\int_{\mathbf{R}^{n}} \exp (i\langle t-s, x\rangle) a(\omega, x-\nu) \hat{g}(s) d s\right] d x\right] \\
&=\int_{\mathbf{R}^{n}}\left[( 2 \pi ) ^ { - n } \int _ { \widehat { \mathbf { R } } ^ { n } } \left[\int_{\mathbf{R}^{n}} \exp (i\langle t, \gamma\rangle) \eta\left(\alpha_{-t}(\omega)\right) d t\right.\right. \\
&=\int_{\mathbf{R}^{n}}\left[( 2 \pi ) ^ { - n } \int _ { \widehat { \mathbf { R } } ^ { n } } \left[\int_{\mathbf{R}^{n}} \exp (i\langle t-s, x\rangle) a(\omega, x-\nu-\gamma)\right.\right. \\
&\left.\left.\times a(\omega, x-\nu-\gamma) \hat{g}^{\gamma}(s) d s\right] d x\right] \eta\left(\alpha_{-t}(\omega)\right) d t \\
&=\left(A^{\nu+\gamma}\left(\hat{g}^{\gamma} * \eta\right)\right)(\omega),
\end{aligned}
$$

where

$$
\hat{g}^{\gamma}(s)=\exp (i\langle s, \gamma\rangle) \hat{g}(s) .
$$

Since $g=1$ on $B_{S}=B_{1+|\gamma|+R}$, the function

$$
g^{\gamma}(\lambda)=\int_{\mathbf{R}^{n}} \hat{g}^{\gamma}(s) \exp (-i\langle s, \lambda\rangle) d s=g(\lambda-\gamma)
$$

equals 1 on $B_{1+R}$. This implies $\hat{g}^{\gamma} * \eta=\eta$. Therefore

$$
\Phi^{\gamma}\left(A^{\nu}\right) \eta=\Phi^{\gamma}(T) \eta=A^{\nu+\gamma}\left(\hat{g}^{\gamma} * \eta\right)=A^{\nu+\gamma} \eta \text {. }
$$

(ii) Let $T, \eta$, and $\psi$ be as above. According to Lemma 3.1(ii),

$$
\begin{aligned}
\left\langle\Phi^{\gamma}\left(B A^{\nu}\right) \eta, \varphi\right\rangle & =L\left(\left\{\sum_{j=1}^{p_{k}}\left\langle B A^{\nu} f_{k, j}^{\gamma} \eta, f_{k, j}^{\gamma} \varphi\right\rangle\right\}_{k=1}^{\infty}\right) \\
& =L\left(\left\{\sum_{j=1}^{p_{k}}\left\langle B T f_{k, j}^{\gamma} \eta, f_{k, j}^{\gamma} \varphi\right\rangle\right\}_{k=1}^{\infty}\right)=\left\langle\Phi^{\gamma}(B T) \eta, \varphi\right\rangle \\
& =\left\langle\Phi^{\gamma}(B) \Phi^{\gamma}(T) \eta, \psi\right\rangle=\left\langle\Phi^{\gamma}(B) A^{\nu+\gamma} \eta, \psi\right\rangle .
\end{aligned}
$$

Proof of Theorem 1. (i) Suppose that $S$ is a bounded operator on $L^{2}\left(\Omega \times\left(\mathbf{R}^{n} / \Gamma\right)\right)$ such that $S(A-z)=1$. It follows from (2.3) that

$$
(1 \otimes \mathscr{F}) S(1 \otimes \mathscr{F})^{-1}\left[\int_{\Gamma^{\prime}} \bigoplus\left(A^{\gamma}-z\right) d m(\gamma)\right]=1
$$

on $L^{2}\left(\Omega \times \Gamma^{\prime}\right)$. Fix a $\gamma_{0} \in \Gamma^{\prime}$. Let $\eta \in \mathscr{S}_{0}(\Omega)$ such that $\int_{\Omega}|\eta|^{2} d \mu=1$. Then $\gamma \mapsto A^{\gamma} \eta$ is a continuous map from $\Gamma^{\prime}$ into $L^{2}(\Omega)$ (see the definition of $A^{\gamma}$ in $\S 2)$. Let $E_{n}=\left\{\gamma \in \Gamma^{\prime}:\left|\gamma-\gamma_{0}\right| \leq 1 / n\right\}$. Then $\eta \chi_{E_{n}} / \sqrt{m\left(E_{n}\right)}$ is a unit vector 
in $L^{2}\left(\Omega \times \Gamma^{\prime}\right)$. It follows from (3.4) that

$$
\begin{aligned}
1 & =\left\|(1 \otimes \mathscr{F}) S(1 \otimes \mathscr{F})^{-1}\left[\int_{\Gamma^{\prime}} \bigoplus\left(A^{\gamma}-z\right) d m(\gamma)\right] \eta \chi_{E_{n}} / \sqrt{m\left(E_{n}\right)}\right\|_{2}^{2} \\
& \leq\|S\|^{2}\left\|\left[\int_{\Gamma^{\prime}} \bigoplus\left(A^{\gamma}-z\right) d m(\gamma)\right] \eta \chi_{E_{n}} / \sqrt{m\left(E_{n}\right)}\right\|_{2}^{2} \\
& =\|S\|^{2} \frac{1}{m\left(E_{n}\right)} \int_{E_{n}}\left(\int_{\Omega}\left|\left(\left(A^{\gamma}-z\right) \eta\right)(\omega)\right|^{2} d \mu(\omega)\right) d m(\gamma) .
\end{aligned}
$$

Letting $n \rightarrow \infty$, we see that

$$
1 /\|S\|^{2} \leq\left\|\left(A^{\gamma_{0}}-z\right) \eta\right\|_{2}^{2} .
$$

This shows that $z \notin \sigma_{l}\left(A^{\gamma_{0}}\right)$. Hence we have shown that $\sigma_{l}(A) \supset \sigma_{l}\left(A^{\gamma}\right)$ for every $\gamma \in \Gamma^{\prime}$.

On the other hand, if $z \notin \sigma_{l}\left(A^{\nu}\right)$, then there is a bounded operator $B$ on $L^{2}(\Omega)$ such that $B\left(A^{\nu}-z\right)=1$. By Lemma 3.2(ii), $\Phi^{\gamma}(B)\left(A^{\nu+\gamma}-z\right)=$ $\Phi^{\gamma}(B) A^{\nu+\gamma}-z \Phi^{\gamma}(B)=\Phi^{\gamma}\left(B\left(A^{\nu}-z\right)\right)=1$ for every $\gamma \in \Gamma^{\prime}$. Hence for any $\eta \in \mathscr{S}_{0}(\Omega)$, we have

$$
\|\eta\|_{2}=\left\|\Phi^{\gamma}(B)\left(A^{\nu+\gamma}-z\right) \eta\right\|_{2} \leq\|B\|\left\|\left(A^{\nu+\gamma}-z\right) \eta\right\|_{2}
$$

for every $\gamma \in \Gamma^{\prime}$. It follows from this inequality and (2.3) that

$$
\|\xi\|_{2} /\|B\| \leq\|(A-z) \xi\|_{2}
$$

for every $\xi$ in the domain of $A$. Hence $z \notin \sigma_{l}(A)$. Thus we have shown $\sigma_{l}(A) \subset \sigma_{l}\left(A^{\nu}\right)$ for every $\nu \in \Gamma^{\prime}$.

(ii) It follows from Lemma 3.2(i) that if one $A^{\gamma}$ is bounded, then every $A^{\gamma}$ is bounded. On the other hand, by (2.3), $A$ is bounded if and only if $A^{\gamma}$ is bounded for almost every $\gamma$.

(iii) If $A$ is bounded, the pseudo-differential operator $B=A^{*} A$ is selfadjoint. It is easy to see that $B^{\gamma}=\left(A^{\gamma}\right)^{*} A^{\gamma}$. By (i), therefore, we have $\sigma(B)=\sigma_{l}(B)=\sigma_{l}\left(B^{\gamma}\right)=\sigma\left(B^{\gamma}\right)$. This implies $\|A\|^{2}=\|B\|=\left\|B^{\gamma}\right\|=\left\|A^{\gamma}\right\|^{2}$.

(iv) If $A$ is bounded, we can apply Lemmas 3.1 and 3.2 to $A^{*}$ and $\left(A^{\gamma}\right)^{*}$, since these operators are now closures of bounded pseudo-differential operators induced by their common symbol function $a^{*}$ on the respective $\mathscr{S}_{0}$-spaces. Hence, by $(\mathrm{i}), \sigma_{r}(A)=\sigma_{l}\left(A^{*}\right)=\sigma_{l}\left(\left(A^{\gamma}\right)^{*}\right)=\sigma_{r}\left(A^{\gamma}\right)$.

Proof of Theorem 2. Suppose that $A$ is symmetric. Let $\eta \in \mathscr{S}_{0}(\Omega)$ and $f \in$ $\mathscr{S}_{0}\left(\mathbf{R}^{n} / \Gamma\right)$. Then $\eta f$ belongs to the domain of $A$. It follows from (2.3) that

$$
\int_{\Gamma^{\prime}}\left\langle A^{\gamma} \eta, \eta\right\rangle|(\mathscr{F} f)(\gamma)|^{2} d m(\gamma)=\langle A \eta f, \eta f\rangle \in \mathbf{R} .
$$

Hence $\left\langle A^{\gamma} \eta, \eta\right\rangle \in \mathbf{R}$ for almost every $\gamma \in \Gamma^{\prime}$. Since the map $\gamma \mapsto\left\langle A^{\gamma} \eta, \eta\right\rangle$ is continuous (see $\S 2$ ), $\left\langle A^{\gamma} \eta, \eta\right\rangle \in \mathbf{R}$ for every $\gamma \in \Gamma^{\prime}$. Hence if $A$ is symmetric, then so is every $A^{\gamma}$. Conversely, it follows from (2.3) that if $A^{\gamma}$ is symmetric for almost every $\gamma \in \Gamma^{\prime}$, then $A$ is symmetric. Therefore (ii) and (iii) are equivalent. If we take the identity (2.2) and apply a similar argument, we see that (iii) and (iv) are equivalent. Obviously (ii) implies (i). On the other hand, that (i) implies (ii) follows from Lemma 3.2(i).

We will now turn our attention to the proof of Theorem 5 . Recall that, in a natural way, the space $L^{2}\left(\Omega \times\left(\mathbf{R}^{n} / \Gamma\right)\right)$ is identified with $L^{2}\left(\mathbf{R}^{n} / \Gamma, L^{2}(\Omega)\right)$, 
the collection of $L^{2}(\Omega)$-valued $L^{2}$-functions on $\mathbf{R}^{n} / \Gamma$. Let $\mathscr{D}$ denote the collection of functions in $L^{\infty}\left(\mathbf{R}^{n} / \Gamma, L^{\infty}(\Omega)\right.$ ) whose supports are bounded in $\mathbf{R}^{n} / \Gamma$. Obviously $\mathscr{D}$ is a dense linear subspace in $L^{2}\left(\mathbf{R}^{n} / \Gamma, L^{2}(\Omega)\right)$. Since $u_{t}=1$ for every $t \in \Gamma$, we may use the expression $u_{s}$ for $s \in \mathbf{R}^{n} / \Gamma$. Let $\mathscr{C}_{2}$ denote the collection of bounded linear operators $K$ on $L^{2}\left(\Omega \times\left(\mathbf{R}^{n} / \Gamma\right)\right)=$ $L^{2}\left(\mathbf{R}^{n} / \Gamma, L^{2}(\Omega)\right)$ which has a kernel function $k \in L^{2}\left(\mathbf{R}^{n} / \Gamma, L^{2}(\Omega)\right)$ in the sense that

$$
(K \xi)(t)=\int_{\mathbf{R}^{n} / \Gamma} k(s) u_{-s} \xi(t-s) d \tau(s)
$$

for every $\xi \in \mathscr{D}$. If $K \in \mathscr{C}_{2}$, then $K^{*} \in \mathscr{C}_{2}$. Indeed it is straightforward to verify that $K^{*}$ has $k^{*}(s)=u_{-s} \overline{k(-s)}$ as its kernel function. Furthermore, for $\zeta \in L^{2}\left(\mathbf{R}^{n} / \Gamma, L^{2}(\Omega)\right)$ and $\xi \in \mathscr{D}$,

$$
\langle K \zeta, \xi\rangle=\left\langle\zeta, K^{*} \xi\right\rangle=\int_{\mathbf{R}^{n} / \Gamma}\left\langle\zeta(t), \int_{\mathbf{R}^{n} / \Gamma} k^{*}(s) u_{-s} \xi(t-s) d \tau(s)\right\rangle_{\mu} d \tau(t) .
$$

(Here, to avoid ambiguity, we use $\langle\cdot, \cdot\rangle_{\mu}$ to denote the inner product on $L^{2}(\Omega)$.) Since the function $t \mapsto \int_{\mathbf{R}^{n} / \Gamma}\left|k^{*}(s) u_{-s} \xi(t-s)\right| d \tau(s)$ belongs to $L^{2}\left(\mathbf{R}^{n} / \Gamma, L^{2}(\Omega)\right)$,

$$
(t, s) \mapsto \zeta(t) \overline{k^{*}(s)} u_{-s} \overline{\xi(t-s)}
$$

is an $L^{1}(\Omega)$-valued $L^{1}$-function with respect to the measure $\tau \times \tau$. The operators $\left\{u_{s}: s \in \mathbf{R}^{n} / \Gamma\right\}$ are also isometries on $L^{1}(\Omega)$ in the obvious way. With this in mind, we define

$$
(V F)(s, t)=u_{-s} F(-s, t-s)
$$

for $F \in L^{1}\left(\left(\mathbf{R}^{n} / \Gamma\right) \times\left(\mathbf{R}^{n} / \Gamma\right), L^{1}(\Omega)\right.$.) Then $V$ is an isometry on that $L^{1}$-space and preserves the integral. If we set $F(s, t)=\zeta(t) \overline{k^{*}(s)} u_{-s} \overline{\xi(t-s)}$, then

$$
(V F)(s, t)=k(s) \overline{\xi(t)} u_{-s} \zeta(t-s) .
$$

Hence, if we set

$$
G(t)=\int_{\mathbf{R}^{n} / \Gamma} k(s) u_{-s} \zeta(t-s) d \tau(s),
$$

then by Fubini's theorem,

$$
\langle K \zeta, \xi\rangle=\int_{\mathbf{R}^{n} / \Gamma}\langle G(t), \xi(t)\rangle_{\mu} d \tau(t) .
$$

Since $G$ belongs to $L^{1}\left(E, L^{1}(\Omega)\right)$ for every bounded measurable set $E \subset$ $\mathbf{R}^{n} / \Gamma$ and $\xi$ is an arbitrary element in $\mathscr{D}$, the above inequality implies that $\left[\int_{E}\|G(t)\|_{\mu, 2}^{2} d \tau(t)\right]^{1 / 2} \leq\|K \zeta\|_{2}$. Since $E$ is arbitrary, $G \in L^{2}\left(\mathbf{R}^{n} / \Gamma, L^{2}(\Omega)\right)$. This means $\langle K \zeta, \xi\rangle=\langle G, \xi\rangle$. Hence we have established the following:

Lemma 3.3. Suppose that $k$ is the kernel function for $K \in \mathscr{C}_{2}$ and

$$
\zeta \in L^{2}\left(\Omega \times\left(\mathbf{R}^{n} / \Gamma\right)\right)=L^{2}\left(\mathbf{R}^{n} / \Gamma, L^{2}(\Omega)\right) .
$$

Then

$$
(K \zeta)(t)=\int_{\mathbf{R}^{n} / \Gamma} k(s) u_{-s} \zeta(t-s) d \tau(s)
$$

Let $\mathscr{N}$ be the closure of $\mathscr{C}_{2}$ in the weak operator topology. 
Lemma 3.4. $\mathscr{C}_{2}$ is an ideal in $\mathscr{N}$. More precisely, if $k$ is the kernel function for $K \in \mathscr{C}_{2}$, then for any $T \in \mathcal{N}, T k$ is the kernel function for $T K$.

Proof. Suppose that $K \in \mathscr{C}_{2}$ with kernel $k$, and suppose that $H \in \mathscr{C}_{2}$. Let $\xi, \eta \in \mathscr{D}$. Define $[\xi \eta](s)=\int_{\mathbf{R}^{n} / \Gamma} \eta(t) u_{-s} \overline{\xi(t-s)} d \tau(t)$. With Lemma 3.3 in mind, it is easy to verify that $\langle H K \xi, \eta\rangle=\langle H k,[\xi \eta]\rangle$. This shows that $H K$ belongs to $\mathscr{C}_{2}$ and has $H k$ as its kernel function. Suppose now that $T$ is an operator in $\mathscr{N}$. Then there is a sequence $\left\{H_{n}\right\} \in \mathscr{C}_{2}$ such that $\lim _{n \rightarrow \infty}\left\langle H_{n} k,[\xi \eta]\right\rangle=\langle T k,[\xi \eta]\rangle$ and $\lim _{n \rightarrow \infty}\left\langle H_{n} K \xi, \eta\right\rangle=\langle T K \xi, \eta\rangle$. Since $\left\langle H_{n} K \xi, \eta\right\rangle=\left\langle H_{n} k,[\xi \eta]\right\rangle$, we have $\langle T K \xi, \eta\rangle=\langle T k,[\xi \eta]\rangle$. It follows from Fubini's theorem that

$$
\begin{aligned}
\langle T K \xi, \eta\rangle & =\langle T k,[\xi \eta]\rangle \\
& =\int_{\mathbf{R}^{n} / \Gamma} \int_{\mathbf{R}^{n} / \Gamma}\left\langle(T k)(s) u_{-s} \xi(t-s), \eta(t)\right\rangle_{\mu} d \tau(s) d \tau(t) .
\end{aligned}
$$

Since $\xi \in \mathscr{D}$, the function $t \mapsto \int_{\mathbf{R}^{n} / \Gamma}(T k)(s) u_{-s} \xi(t-s) d \tau(s)$ belongs to $L^{2}\left(\mathbf{R}^{n} / \Gamma, L^{2}(\Omega)\right)$. Hence $(T K \xi)(t)=\int_{\mathbf{R}^{n} / \Gamma}(T k)(s) u_{-s} \xi(t-s) d \tau(s)$. Therefore $T k$ is the kernel function for $T K$.

For each $s \in \mathbf{R}^{n}$, let $\widetilde{U}_{s}$ be the unitary operator

$$
\left(\tilde{U}_{s} \zeta\right)(\omega, t+\Gamma)=\zeta\left(\alpha_{-s}(\omega), t-s+\Gamma\right)
$$

on $L^{2}\left(\Omega \times\left(\mathbf{R}^{n} / \Gamma\right)\right)$.

Lemma 3.5. (i) Let $f \in \mathscr{S}\left(\mathbf{R}^{n}\right)$. Then the operator

$$
(F \zeta)(\omega, t+\Gamma)=\int_{\mathbf{R}^{n}} f(s) \zeta\left(\alpha_{-s}(\omega), t-s+\Gamma\right) d s, \quad \zeta \in L^{2}\left(\mathbf{R}^{n} / \Gamma, L^{2}(\Omega)\right),
$$

belongs to $\mathscr{C}_{2}$.

(ii) Suppose that $g$ is a measurable function $\Omega \times \mathbf{R}^{n}$ such that $\int_{\mathbf{R}^{n}}\|g(\cdot s)\|_{\infty} d s$ $<\infty$. Then the operator $G: \mathscr{S}_{0}\left(\Omega \times\left(\mathbf{R}^{n} / \Gamma\right)\right) \rightarrow L^{2}\left(\Omega \times\left(\mathbf{R}^{n} / \Gamma\right)\right)$ defined by the formula

$$
(G \zeta)(\omega, t+\Gamma)=\int_{\mathbf{R}^{n}} g(\omega, s) \zeta\left(\alpha_{-s}(\omega), t-s+\Gamma\right) d s
$$

extends to a bounded operator on $L^{2}\left(\Omega \times\left(\mathbf{R}^{n} / \Gamma\right)\right)$ which belongs to $\mathcal{N}$.

Proof. (i) Let

$$
\widetilde{U}_{s}=\int_{\widehat{\mathbf{R}}^{n}} \exp (i\langle s, \gamma\rangle) d \widetilde{E}_{\gamma}
$$

be the spectral decomposition of the unitary group $\left\{\widetilde{U}_{s}: s \in \mathbf{R}^{n}\right\}$. Since $\widetilde{U}_{s}=$ 1 for every $s \in \Gamma$, the spectral measure $d \widetilde{E}_{\gamma}$ is actually supported on $\Gamma^{\prime}$. It is easy to see that

$$
F=\int_{\mathbf{R}^{n}} f(s) \widetilde{U}_{s} d s=\int_{\widehat{\mathbf{R}}^{n}} \hat{f}(\gamma) d \widetilde{E}_{\gamma}=\int_{\Gamma^{\prime}} \hat{f}(\gamma) d \widetilde{E}_{\gamma},
$$

where $\hat{f}$ is the standard Fourier transform of $f$ and, therefore, belongs to $\mathscr{S}\left(\widehat{\mathbf{R}}^{n}\right)$. Consider $\hat{f}$ as a function on $\Gamma^{\prime}$. Recall that $\mathscr{F}$ denotes the Fourier transform from $L^{2}\left(\mathbf{R}^{n} / \Gamma\right)$ to $L^{2}\left(\Gamma^{\prime}\right)$. We also have

$$
F=\int_{\mathbf{R}^{n} / \Gamma}\left(\mathscr{F}^{-1} \hat{f}\right)(s+\Gamma) \tilde{U}_{s} d \tau(s+\Gamma) .
$$


That is,

$$
(F \zeta)(\omega, t+\Gamma)=\int_{\mathbf{R}^{n} / \Gamma}\left(\mathscr{F}^{-1} f\right)(s+\Gamma) u_{-s} \zeta(t-s+\Gamma) d \tau(s+\Gamma)
$$

for $\zeta \in L^{2}\left(\mathbf{R}^{n} / \Gamma, L^{2}(\Omega)\right)$. Hence $F \in \mathscr{C}_{2}$.

(ii) First of all, it is trivial that $G$ extends to a bounded operator on $L^{2}\left(\Omega \times\left(\mathbf{R}^{n} / \Gamma\right)\right)$ and that

$$
\|G\| \leq \int_{\mathbf{R}^{n}}\|g(\cdot, s)\|_{\infty} d s
$$

It follows from (3.5) that if $g(\omega, s)=\varphi(\omega) f(s)$ with $\varphi \in L^{\infty}(\Omega)$ and $f \in$ $\mathscr{S}\left(\mathbf{R}^{n}\right)$, then $G \in \mathscr{C}_{2}$. For an arbitrary $g$, there is an $M>0$ such that for any $\varepsilon>0$, there is a $g_{\varepsilon}=\sum_{j=1}^{k} \varphi_{j} f_{j}$ with $\varphi_{j} \in L^{\infty}(\Omega)$ and $f_{j} \in \mathscr{S}_{0}\left(\mathbf{R}^{n}\right)$ which satisfies

$$
\int_{\mathbf{R}^{n}}\left\|g_{\varepsilon}(\cdot, s)\right\|_{\infty} d s \leq M
$$

and

$$
\int_{\mathbf{R}^{n}} \int_{\Omega}\left|g_{\varepsilon}(\omega, s)-g(\omega, s)\right| d \mu(\omega) d s \leq \varepsilon .
$$

Each $g_{\varepsilon}$ generates an operator $G_{\varepsilon} \in \mathscr{C}_{2}$ by the formula

$$
\left(G_{\varepsilon} \zeta\right)(\omega, t+\Gamma)=\int_{\mathbf{R}^{n}} g_{\varepsilon}(\omega, s) \zeta\left(\alpha_{-s}(\omega), t-s+\Gamma\right) d s .
$$

Clearly, $\lim _{\varepsilon \rightarrow 0} G_{\varepsilon}=G$ in the weak operator topology.

For each natural number $k$, let $0 \leq h_{k} \leq 1$ be a $C^{\infty}$-function on $[0, \infty)$ such that $h_{k}=1$ on $[0, k]$ and $h_{k}=0$ on $[k+1, \infty)$. Then $h_{k}(|\gamma|)$ is a compactly supported $C^{\infty}$-function on $\widehat{\mathbf{R}^{n}}$. Let

$$
g_{k}(t)=(2 \pi)^{-n / 2} \int_{\widehat{\mathbf{R}}^{n}} \exp (-i\langle t, \gamma\rangle) h_{k}(|\gamma|) d \gamma .
$$

Then $g_{k} \in \mathscr{S}\left(\mathbf{R}^{n}\right)$ and

$$
h_{k}(|\gamma|)=(2 \pi)^{-n / 2} \int_{\mathbf{R}^{n}} \exp (i\langle t, \gamma\rangle) g_{k}(t) d t .
$$

Define

It is obvious that

$$
H_{k}=\int_{\widehat{\mathbf{R}}^{n}} h_{k}(|\gamma|) d \widetilde{E}_{\gamma}
$$

$$
H_{k}=(2 \pi)^{-n / 2} \int_{\mathbf{R}^{n}} g_{k}(s) \widetilde{U}_{s} d s
$$

That is,

$$
\left(H_{k} \zeta\right)(\omega, t+\Gamma)=(2 \pi)^{-n / 2} \int_{\mathbf{R}^{n}} g(s) \zeta\left(\alpha_{-s}(\omega), t-s+\Gamma\right) d s
$$

for $\zeta \in L^{2}\left(\Omega \times\left(\mathbf{R}^{n} / \Gamma\right)\right)$. By Lemma 3.5(i), $H_{k} \in \mathscr{C}_{2}$.

For the rest of the section, the symbol function $a$ of the pseudo-differential operator $A$ will be assumed to be elliptic and formally selfadjoint. 
Lemma 3.6. For any $\varphi \in C_{0}(\mathbf{R}), \varphi(A) \in \mathscr{N}$. Consequently for any interval $I \subset \mathbf{R}, \chi_{I}(A) \in \mathscr{N}$.

Proof. Let us first show that for any $\eta \in \mathscr{S}_{0}(\Omega \times(\mathbf{R} / \Gamma))$,

$$
\lim _{k \rightarrow \infty}\left\|A \eta-H_{k} A H_{k} \eta\right\|_{2}=0 .
$$

Since the sequence $\left\{H_{k}\right\}$ strongly converges to the identity operator, it suffices to show that $\lim _{k \rightarrow \infty}\left\|A \eta-A H_{k} \eta\right\|_{2}=0$. Suppose that

$$
\eta(\omega, t+\Gamma)=(f * \zeta)(\omega, t+\Gamma)=\int_{\mathbf{R}^{n}} f(s) \zeta\left(\alpha_{-s}(\omega), t-s+\Gamma\right) d s,
$$

where $f \in \mathscr{S}_{0}\left(\mathbf{R}^{n}\right)$ and $\zeta \in L^{2}\left(\Omega \times\left(\mathbf{R}^{n} / \Gamma\right)\right) \cap L^{\infty}\left(\Omega \times\left(\mathbf{R}^{n} / \Gamma\right)\right)$. Then

$$
\begin{aligned}
\left(H_{k} \eta\right) & (\omega, t+\Gamma) \\
= & (2 \pi)^{-n / 2} \int_{\mathbf{R}^{n}} g_{k}(r)\left[\int_{\mathbf{R}^{n}} f(s) \zeta\left(\alpha_{-s-r}(\omega), t-s-r+\Gamma\right) d s\right] d r \\
= & \int_{\mathbf{R}^{n}} f(s)\left[(2 \pi)^{-n / 2} \int_{\mathbf{R}^{n}} g_{k}(r) \zeta\left(\alpha_{-s-r}(\omega), t-s-r+\Gamma\right) d r\right] d s \\
= & \left(f *\left(H_{k} \zeta\right)\right)(\omega, t+\Gamma) .
\end{aligned}
$$

Hence by the definition of the operator $A$, we have

$$
\begin{aligned}
\left(A H_{k} \eta\right)(\omega, y+\Gamma) & =\left(A\left(f *\left(H_{k} \zeta\right)\right)\right)(\omega, y+\Gamma) \\
& =\int_{\mathbf{R}^{n}}\left(a_{\omega}^{0} * f\right)(t)\left(H_{k} \zeta\right)\left(\alpha_{-t}(\omega), y-t+\Gamma\right) d t
\end{aligned}
$$

where

$$
\left(a_{\omega}^{0} * f\right)(t)=(2 \pi)^{-n} \int_{\widehat{\mathbf{R}}^{n}}\left[\int_{\mathbf{R}^{n}} \exp (i\langle t-s, x\rangle) a(\omega, x) f(s) d s\right] d x .
$$

Since $f \in \mathscr{S}_{0}\left(\mathbf{R}^{n}\right)$ and $a \in S^{m}$, it follows from Lemma 3.5(ii) that $a_{\omega}^{0} * f$ is the kernel function of a bounded operator in $\mathscr{N}$. Because $\lim _{k \rightarrow \infty}\left\|H_{k} \zeta-\zeta\right\|_{2}=0$, we have $\lim _{k \rightarrow \infty}\left\|A H_{k} \eta-A \eta\right\|_{2}=\lim _{k \rightarrow \infty}\left\|A\left(f *\left(H_{k} \zeta\right)\right)-A(f * \zeta)\right\|_{2}=0$.

Since $H_{k}(f * \zeta)=(2 \pi)^{-n / 2} g_{k} *(f * \zeta)$, we also have

$$
\left(A H_{k}(f * \zeta)\right)(\omega, y)=(2 \pi)^{-n / 2} \int_{\mathbf{R}^{n}}\left(a_{\omega}^{0} * g_{k}\right)(t)(f * \zeta)\left(\alpha_{-t}(\omega), y-t+\Gamma\right) d t .
$$

Because $g_{k} \in \mathscr{S}\left(\mathbf{R}^{n}\right)$ and $a \in S^{m}$, by Lemma 3.5(ii), the function $(\omega, t)$ $\mapsto\left(a_{\omega}^{0} * g_{k}\right)(t)$ is the kernel of a bounded operator in $\mathscr{N}$. That is, $A H_{k}$ extends to a bounded operator which belongs to $\mathscr{N}$. Hence if $z \in \mathbf{C} \backslash \mathbf{R}$, then $\left(H_{k} A H_{k}-z\right)^{-1} \in \mathscr{N}$. To complete the proof, it suffices to show that $(A-z)^{-1} \in$ $\mathscr{N}$ for every $z \in \mathbf{C} \backslash \mathbf{R}$. For any $\eta \in \mathscr{S}_{0}\left(\Omega \times\left(\mathbf{R}^{n} / \Gamma\right)\right)$, we have

$$
\begin{aligned}
\|[(A & \left.-z)^{-1}-\left(H_{k} A H_{k}-z\right)^{-1}\right](A-z) \eta \|_{2} \\
& =\left\|\eta-\left(H_{k} A H_{k}-z\right)^{-1}\left(H_{k} A H_{k}-z+A-H_{k} A H_{k}\right) \eta\right\|_{2} \\
& =\left\|\left(H_{k} A H_{k}-z\right)^{-1}\left(A-H_{k} A H_{k}\right) \eta\right\|_{2} \\
& \leq|\operatorname{Im} z|^{-1}\left\|\left(A-H_{k} A H_{k}\right) \eta\right\|_{2} .
\end{aligned}
$$

Since $A$ is selfadjoint and $\mathscr{S}_{0}\left(\Omega \times\left(\mathbf{R}^{n} / \Gamma\right)\right)$ is a core for $A$, we have that $(A-z) \mathscr{S}_{0}\left(\Omega \times\left(\mathbf{R}^{n} / \Gamma\right)\right)$ is dense in $L^{2}\left(\Omega \times\left(\mathbf{R}^{n} / \Gamma\right)\right)$. Therefore the above 
estimate implies that $(A-z)^{-1}$ is the strong limit of $\left\{\left(H_{k} A H_{k}-z\right)^{-1}\right\}$. Hence $(A-z)^{-1} \in \mathscr{N}$ if $z \in \mathbf{C} \backslash \mathbf{R}$.

Lemma 3.7. Let

$$
\tilde{g}_{k}(t+\Gamma)=\int_{\Gamma^{\prime}} \exp (-i\langle t, \gamma\rangle) h_{k}(|\gamma|) d m(\gamma) .
$$

Suppose that $H \in \mathscr{N}$, and suppose that $H \tilde{g}_{k}=0$ for every $k$. Then $H=0$.

Proof. Let $U_{s}$ denote the unitary operator $\left(U_{s} f\right)(t+\Gamma)=f(t-s+\Gamma)$ on $L^{2}\left(\mathbf{R}^{n} / \Gamma\right)$. Then the unitary group $\left\{U_{s}: s \in \mathbf{R}^{n}\right\}$ has the spectral decomposition

$$
U_{s}=\int_{\widehat{\mathbf{R}}^{n}} \exp (i\langle s, \gamma\rangle) d E_{\gamma} .
$$

Again, because $U_{s}=1$ for every $s \in \Gamma$, the spectral measure $d E_{\gamma}$ is supported on $\Gamma^{\prime}$. Hence

$$
\int_{\mathbf{R}^{n} / \Gamma} \tilde{g}_{k}(s+\Gamma) U_{s} d \tau(s)=\int_{\Gamma^{\prime}} h_{k}(|\gamma|) d E_{\gamma}=\int_{\widehat{\mathbf{R}}^{n}} h_{k}(|\gamma|) d E_{\gamma} .
$$

Since $h_{k}(|\gamma|) \rightarrow 1$ for every $\gamma$, the sequence $\left\{\int_{\mathbf{R}^{n} / \Gamma} \tilde{g}_{k}(s+\Gamma) U_{s} d \tau(s)\right\}$ converges to the identity operator strongly.

To prove the lemma, let us first assume that $H \in \mathscr{C}_{2}$ and that $h$ is its kernel function. We have

$$
\begin{aligned}
\left(H \tilde{g}_{k}\right)(\omega, t+\Gamma) & =\int_{\mathbf{R}^{n} / \Gamma} h(\omega, s+\Gamma) \tilde{g}_{k}(t-s+\Gamma) d \tau(s+\Gamma) \\
& =\int_{\mathbf{R}^{n} / \Gamma} \tilde{g}_{k}(s+\Gamma) h(\omega, t-s+\Gamma) d \tau(s+\Gamma) \\
& =\left(\left(1 \otimes\left[\int_{\mathbf{R}^{n} / \Gamma} \tilde{g}_{k}(s+\Gamma) U_{s} d \tau(s+\Gamma)\right]\right) h\right)(\omega, t+\Gamma) .
\end{aligned}
$$

Hence $\lim _{k \rightarrow \infty}\left\|H \tilde{g}_{k}-h\right\|_{2}=0$. If $H \tilde{g}_{k}=0$ for every $k$, then $h=0$.

Suppose now that $H$ is an arbitrary element in $\mathscr{N}$. Since the operators $H_{k}=\int_{\Gamma^{\prime}} h_{k}(|\gamma|) d \widetilde{E}_{\gamma}$ converge to 1 strongly, if $H \neq 0$, there is a $p$ such that $H_{p} H \neq 0$. Since $H_{p} H \in \mathscr{C}_{2}$ (Lemma 3.4), by the previous paragraph, this is possible only if there is a $\tilde{g}_{k}$ such that $H_{p} H \tilde{g}_{k} \neq 0$.

Proof of Theorem 5. Let $I$ be an open interval in $\mathbf{R}$. Then $I \cap \sigma(A) \neq \varnothing$ if and only if $\chi_{I}(A) \neq 0$. By Lemmas 3.6 and 3.7, this happens precisely when there is a $k$ such that $\left\langle\chi_{I}(A) \tilde{g}_{k}, \tilde{g}_{k}\right\rangle>0$. Let $\nu_{k}$ be the measure $\nu_{k}((-\infty, t))=$ $\left\langle\chi_{(-\infty, t)}(A) \tilde{g}_{k}, \tilde{g}_{k}\right\rangle$. Then $I \cap \sigma(A) \neq \varnothing$ if and only if $\nu_{k}(I)>0$ for some $k$ : So the proof will be complete once we show $V_{R}(I) \leq \nu_{k}(I) \leq V_{R^{\prime}}(I)$ for $R<k<R^{\prime}-1$.

We have

$$
\left\langle(A-z)^{-1} \tilde{g}_{k}, \tilde{g}_{k}\right\rangle=\left\langle(1 \otimes \mathscr{F})(A-z)^{-1}(1 \otimes \mathscr{F})^{-1}(1 \otimes \mathscr{F}) \tilde{g}_{k},(1 \otimes \mathscr{F}) \tilde{g}_{k}\right\rangle .
$$

Since $\left((1 \otimes \mathscr{F}) \tilde{g}_{k}\right)(\omega, \gamma)=\left(\mathscr{F} \tilde{g}_{k}\right)(\gamma)=h_{k}(|\gamma|)$, it follows from (2.3) that

$$
\begin{aligned}
\int_{\mathbf{R}} \frac{1}{t-z} d \nu_{k}(t) & =\left\langle(A-z)^{-1} \tilde{g}_{k}, \tilde{g}_{k}\right\rangle \\
& =\int_{\Gamma^{\prime}}\left\langle\left(A^{\gamma}-z\right)^{-1} 1,1\right\rangle\left(h_{k}(|\gamma|)\right)^{2} d m(\gamma) .
\end{aligned}
$$


Suppose that $z=x+i y$ lies in the upper half-plane. Then

$$
\begin{aligned}
\int_{\mathbf{R}} \frac{y}{(t-x)^{2}+y^{2}} d \nu_{k}(t) \\
\quad=\frac{1}{2 i}\left[\int_{\mathbf{R}} \frac{1}{t-z} d \nu_{k}(t)-\int_{\mathbf{R}} \frac{1}{t-\bar{z}} d \nu_{k}(t)\right] \\
\quad=\frac{1}{2 i}\left\langle\left[(A-z)^{-1}-(A-\bar{z})^{-1}\right] \tilde{g}_{k}, \tilde{g}_{k}\right\rangle \\
\quad=\int_{\Gamma^{\prime}}\left\langle\frac{1}{2 i}\left[\left(A^{\gamma}-z\right)^{-1}-\left(A^{\gamma}-\bar{z}\right)^{-1}\right] 1,1\right\rangle\left(h_{k}|\gamma|\right)^{2} d m(\gamma) \\
\quad=\int_{\Gamma^{\prime}} y\left\langle\left(\left(A^{\gamma}-x\right)^{2}+y^{2}\right)^{-1} 1,1\right\rangle\left(h_{k}|\gamma|\right)^{2} d m(\gamma) .
\end{aligned}
$$

Similarly, we have

$$
\int_{\mathbf{R}} \frac{y}{(t-x)^{2}+y^{2}} d V_{R}(t)=\int_{\Gamma^{\prime}} y\left\langle\left(\left(A^{\gamma}-x\right)^{2}+y^{2}\right)^{-1} 1,1\right\rangle \chi_{B_{R}}(\gamma) d m(\gamma) .
$$

Since $\chi_{B_{R}}(\gamma)<\left(h_{k}(|\gamma|)\right)^{2}<\chi_{B_{R^{\prime}}}(\gamma)$ for $R<k<R^{\prime}-1$, we have $V_{R}(I) \leq$ $\nu_{k}(I) \leq V_{R^{\prime}}(I)$.

\section{OPERATORS ASSOCIATED WITH ARBITRARY DYNAMICAL SYSTEMS}

In the previous sections, we assumed that the transformation group $\left\{\alpha_{t}: t \in\right.$ $\left.\mathbf{R}^{n}\right\}$ on $(\Omega, \mathscr{B}, \mu)$ has the property that there is a closed subgroup $\Gamma^{\prime} \subset \widehat{\mathbf{R}}^{n}$ such that for any invariant set $\Delta \in \mathscr{B}$ with $\mu(\Delta)>0$, we have $S_{\Delta}=\Gamma^{\prime}$, where $S_{\Delta}$ is the support of the spectral measure of the unitary group $u_{t} f=f \circ \alpha_{t}$ on the subspace $L^{2}(\Delta, d \mu)$ of $L^{2}(\Omega, d \mu)$. As we have mentioned earlier, if $\mu$ is ergodic, then the dynamical system automatically has this property. But obviously $\mu$ need not be ergodic if the dynamical system has this property. From now on we will drop this assumption. In other words, for the rest of the paper, we assume nothing about the measure-preserving transformation group $\left\{\alpha_{t}: t \in \mathbf{R}^{n}\right\}$ on $(\Omega, \mathscr{B}, \mu)$ except that it is strongly continuous, i.e., $t \mapsto f \circ \alpha_{t}$ is a continuous map from $\mathbf{R}^{n}$ into $L^{2}(\Omega)$ for every $f \in L^{2}(\Omega)$. The space $L^{2}(\Omega)$ is still assumed to be separable.

Let $\left(\Omega^{\#}, \mathscr{B}^{\#}, \mu^{\#}\right)$ be the product measure space of denumerable copies of $(\Omega, \mathscr{B}, \mu)$. Define $\alpha_{t}^{\#}\left(\omega_{1}, \omega_{2}, \ldots\right)=\left(\alpha_{t}\left(\omega_{1}\right), \alpha_{t}\left(\omega_{2}\right), \ldots\right)$. Then $\left\{\alpha_{t}^{\#}: t \in\right.$ $\left.\mathbf{R}^{n}\right\}$ in a strongly continuous group of measure-preserving transformations on the space $L^{2}\left(\Omega^{\#}\right)=L^{2}\left(\Omega^{\#}, \mathscr{B}^{\#}, \mu^{\#}\right)$, which is again separable. For each $t \in$ $\mathbf{R}^{n}$, define $u_{t}^{\#} f=f \circ \alpha_{t}^{\#}, f \in L^{2}\left(\Omega^{\#}\right)$. Then $\left\{u_{t}^{\#}: t \in \mathbf{R}^{n}\right\}$ is a strongly continuous group of unitary operators on $L^{2}\left(\Omega^{\#}\right)$. Let

$$
\Gamma=\left\{t \in \mathbf{R}^{n}: \text { for every } f \in L^{2}(\Omega), f \circ \alpha_{t}=f \text { a.e. }\right\} .
$$

Define

$$
\Gamma^{\prime}=\left\{\gamma \in \widehat{\mathbf{R}}^{n}:\langle t, \gamma\rangle \in 2 \pi \mathbf{Z} \text { for every } t \in \Gamma\right\} .
$$

Theorem 4.1. Suppose that

$$
u_{t}^{\#}=\int_{\widehat{\mathbf{R}}^{n}} \exp (i\langle t, \gamma\rangle) d e_{\gamma}^{\#}
$$


is the spectral decomposition of the unitary group $\left\{u_{t}^{\#}: t \in \mathbf{R}^{n}\right\}$. Then the support of the spectral measure de $e_{\gamma}^{\#}$ is $\Gamma^{\prime}$.

Proof. Since $u_{t}^{\#}=1$ for every $t \in \Gamma$, it is clear that the support of $d e_{\gamma}^{\#}$ is contained in $\Gamma^{\prime}$. It remains to be shown that the support of $d e_{\gamma}^{\#}$ contains $\Gamma^{\prime}$. In other words, we must show that if $\varphi \in C_{c}^{\infty}\left(\widehat{\mathbf{R}}^{n}\right)$ and $\int_{\widehat{\mathbf{R}}^{n}} \varphi(\gamma) d e_{\gamma}^{\#}=0$, then $\varphi$ vanishes on $\Gamma^{\prime}$. Let such a $\varphi$ be given, and let $(2 \pi)^{n / 2} \check{\varphi}$ be its inverse Fourier transform. That is,

$$
\varphi(\gamma)=\int_{\mathbf{R}^{n}} \exp (i\langle t, \gamma\rangle) \check{\varphi}(t) d t .
$$

We have

$$
\int_{\mathbf{R}^{n}} \check{\varphi}(t) u_{t}^{\#} d t=\int_{\widehat{\mathbf{R}}^{n}} \varphi(\gamma) d e_{\gamma}^{\#}=0 .
$$

Suppose that $\eta_{1}, \ldots, \eta_{k}, \xi_{1}, \ldots, \xi_{k} \in L^{2}(\Omega)$. Define

$$
\eta\left(\omega_{1}, \ldots, \omega_{k}, \omega_{k+1}, \omega_{k+2}, \ldots\right)=\eta_{1}\left(\omega_{1}\right) \cdots \eta_{k}\left(\omega_{k}\right)
$$

and

$$
\xi\left(\omega_{1}, \ldots, \omega_{k}, \omega_{k+1}, \omega_{k+2}, \ldots\right)=\xi_{1}\left(\omega_{1}\right) \ldots \xi_{k}\left(\omega_{k}\right)
$$

Then

$$
\begin{aligned}
\left(u_{t}^{\#} \xi\right) & \left(\omega_{1}, \ldots, \omega_{k}, \omega_{k+1}, \omega_{k+2}, \ldots\right) \\
& =\xi\left(\alpha_{t}\left(\omega_{1}\right), \ldots, \alpha_{t}\left(\omega_{k}\right), \alpha_{t}\left(\omega_{k+1}\right), \alpha_{t}\left(\omega_{k+2}\right), \ldots\right) \\
& =\xi_{1}\left(\alpha_{t}\left(\omega_{1}\right)\right) \cdots \xi_{k}\left(\alpha_{t}\left(\omega_{k}\right)\right) \\
& =\left(u_{t} \xi_{1}\right)\left(\omega_{1}\right) \cdots\left(u_{t} \xi_{k}\right)\left(\omega_{k}\right) .
\end{aligned}
$$

Hence, by (4.1), we have

$$
\int_{\mathbf{R}^{n}} \check{\varphi}(t)\left\langle u_{t} \xi_{1}, \eta_{1}\right\rangle \cdots\left\langle u_{t} \xi_{k}, \eta_{k}\right\rangle d t=\int_{\mathbf{R}^{n}} \check{\varphi}(t)\left\langle u_{t}^{\#} \xi, \eta\right\rangle d t=0 .
$$

Let $\mathscr{A}$ be the subalgebra of $C_{b}\left(\mathbf{R}^{n}\right)$ generated by the functions $\left\{\left\langle u_{t} f, g\right\rangle\right.$ : $\left.f, g \in L^{2}(\Omega)\right\}$ in the $t$-variable. Because the complex conjugate of $\left\langle u_{t} f, g\right\rangle$ is $\left\langle u_{t} \bar{f}, \bar{g}\right\rangle$, the algebra $\mathscr{A}$ is symmetric. It follows from (4.2) that

$$
\int_{\mathbf{R}^{n}} \check{\varphi}(t) G(t) d t=0
$$

for every $G \in \mathscr{A}$. Suppose that $t-s \notin \Gamma$. We claim that there exist $f, g \in L^{2}(\Omega)$ such that $\left\langle u_{t} f, g\right\rangle \neq\left\langle u_{s} f, g\right\rangle$. For otherwise, we would have $\left\langle u_{t-s} f, g\right\rangle=\left\langle u_{t}\left(u_{-s} f\right), g\right\rangle=\left\langle u_{s}\left(u_{-s} f\right), g\right\rangle=\langle f, g\rangle$ for every pair of functions $f, g \in L^{2}(\Omega)$. This contradicts the definition of $\Gamma$. Therefore the functions in $\mathscr{A}$ separate the points of $\mathbf{R}^{n} / \Gamma$.

Fix a $\gamma_{0} \in \Gamma^{\prime}$, and let $F(t)=\sin \left(\left\langle t, \gamma_{0}\right\rangle\right)$. Let $1>\varepsilon>0$ be given. Let $h$ be the continuous function on $\mathbf{R}$ such that $h(t)=t$ for $-2 \leq t \leq 2, h(t)=-2$ for $t \leq-2$, and $h(t)=2$ for $t \geq 2$. Let $R>0$ be such that

$$
\int_{\mathbf{R}^{n} \backslash B_{R}}|\check{\varphi}(s)| d s \leq \varepsilon / 6,
$$


where $B_{R}=\left\{t \in \mathbf{R}^{n}:|t| \leq R\right\}$. Since $F$ is a $\Gamma$-periodic function, by the StoneWeierstrass approximation theorem, there is a real-valued function $F_{R} \in \mathscr{A}$ such that

$$
\max _{t \in B_{R}}\left|F(t)-F_{R}(t)\right| \leq \frac{\varepsilon}{4+2 \int_{B_{R}}|\check{\varphi}(s)| d s} .
$$

Since $|F| \leq 1$, we have $\left|F_{R}\right| \leq 1+1 / 4<2$ on $B_{R}$. Hence $h \circ F_{R}(t)=F_{R}(t)$ for every $t \in B_{R}$. Therefore

$$
\max _{t \in B_{R}}\left|F(t)-h \circ F_{R}(t)\right| \leq \frac{\varepsilon}{4+2 \int_{B_{R}}|\check{\varphi}(s)| d s} .
$$

Applying the Stone-Weierstrass approximation theorem once more, we see that $h \circ F_{R}$ belongs to the sup-norm closure of $\mathscr{A}$. Hence it follows from (4.3) that

$$
\int_{\mathbf{R}^{n}} h \circ F_{R}(s) \check{\varphi}(s) d s=0 .
$$

Therefore it follows from (4.4), (4.5) and (4.6) that

$$
\begin{aligned}
& \left|\int_{\mathbf{R}^{n}} \sin \left(\left\langle s, \gamma_{0}\right)\right) \check{\varphi}(s) d s\right| \leq \int_{\mathbf{R}^{n}}\left|F(s)-h \circ F_{R}(s)\right||\check{\varphi}(s)| d s \\
& \quad \leq \int_{B_{R}}\left|F(s)-h \circ F_{R}(s)\right||\check{\varphi}(s)| d s+3 \int_{\mathbf{R}^{n} \backslash B_{R}}|\check{\varphi}(s)| d s \leq \varepsilon .
\end{aligned}
$$

Since $\varepsilon \in(0,1)$ is arbitrary, we have $\int_{\mathbf{R}^{n}} \sin \left(\left\langle s, \gamma_{0}\right\rangle\right) \check{\varphi}(s) d s=0$. A similar argument shows that $\int_{\mathbb{R}^{n}} \cos \left(\left\langle s, \gamma_{0}\right\rangle\right) \check{\varphi}(s) d s=0$. Hence

$$
\varphi\left(\gamma_{0}\right)=\int_{\mathbf{R}^{n}} \exp \left(i\left\langle s, \gamma_{0}\right\rangle\right) \check{\varphi}(s) d s=0 .
$$

This shows that $\varphi$ vanishes on $\Gamma^{\prime}$.

Theorem 4.2. Let $D \in \mathscr{B} \#$ be a set such that $\mu^{\#}(D)>0$ and $\mu^{\#}\left(\left[D \backslash \alpha_{t}^{\#}(D)\right]\right)=$ $\mu^{\#}\left(\left[\alpha_{t}^{\#}(D) \backslash D\right]\right)=0$ for every $t \in \mathbf{R}^{n}$. Then the restricted spectral measure $d e_{\gamma}^{*} \mid L^{2}(D)$ also has $\Gamma^{\prime}$ as its support.

To prove this theorem, we need the following simple measure-theoretical lemma. For a measurable function $f$ on the measure space $\left(\Omega^{\#}, \mathscr{B}^{\#}, \mu^{\#}\right)$, define

$$
\left(S_{k} f\right)\left(\omega_{1}^{\prime}, \ldots, \omega_{k}^{\prime}, \omega_{1}, \omega_{2}, \ldots, \omega_{q}, \ldots\right)=f\left(\omega_{1}, \omega_{2}, \ldots, \omega_{q}, \ldots\right)
$$

for $k=1,2,3, \ldots$ It is clear that for every $1 \leq p \leq \infty, S_{k}$ maps $L^{p}\left(\Omega^{\#}\right)$ isometrically to itself.

Lemma 4.3. Let $E \in \mathscr{B}^{\#}$ and $f \in L^{1}\left(\Omega^{\#}\right)$. Then $\lim _{k \rightarrow \infty} \int_{E} S_{k} f d \mu^{\#}=$ $\mu^{\#}(E) \int_{\Omega^{*}} f d \mu^{\#}$.

Proof. First let us consider the case $f \in L^{\infty}(\Omega)$. Let $\mathscr{U}$ be the algebra of sets generated by $A_{1} \times \cdots \times A_{k} \times \Omega \times \Omega \times \Omega \times \cdots$, where $k$ is an arbitrary natural number and $A_{1}, \ldots, A_{k} \in \mathscr{B}$. Let $\varepsilon>0$ be given. By the definition of the product measure $\mu^{\#}$, there is a sequence $\left\{E_{k}\right\} \subset \mathscr{U}$ such that $E_{k} \supset E$ and $\lim _{k \rightarrow \infty} \mu^{\#}\left(E_{k}\right)=\mu^{\#}(E)$. That is, $\lim _{k \rightarrow \infty}\left\|\chi_{E_{k}}-\chi_{E}\right\|_{1}=0$. Hence there is a $p_{0}$ such that

$$
\left|\int_{E} S_{k} f d \mu^{\#}-\int_{E_{p_{0}}} S_{k} f d \mu^{\#}\right| \leq \varepsilon
$$


for every $k$ and $\mu^{\#}\left(E_{p_{0}} \backslash E\right) \leq \varepsilon$. Suppose that $E_{p_{0}}=B \times \Omega \times \Omega \times \cdots$, where $B$ is a subset of the product of $m_{0}$ copies of $\Omega$. Then $\mu^{\#}\left(E_{p_{0}}\right)=\mu \times \cdots \times \mu(B)$. Furthermore, if $k>m_{0}$, then

$$
\begin{aligned}
\int_{E_{p_{0}}} S_{k} f d \mu^{\#} & \\
= & \int_{B}\left[\int_{\Omega \times \Omega \times \cdots} f\left(\omega_{k}, \omega_{k+1}, \ldots\right) d \mu\left(\omega_{m_{0}+1}\right)\right. \\
& \left.\quad \times \cdots \times \mu\left(\omega_{k}\right) \times \mu\left(\omega_{k+1}\right) \times \cdots\right] d \mu\left(\omega_{1}\right) \times \cdots \times \mu\left(\omega_{m_{0}}\right) \\
= & \mu \times \cdots \times \mu(B) \int_{\Omega \times \Omega \times \cdots} f\left(\omega_{k}, \ldots\right) d \mu\left(\omega_{k}\right) \times \mu\left(\omega_{k+1}\right) \times \cdots \\
= & \mu^{\#}\left(E_{p_{0}}\right) \int_{\Omega^{*}} f d \mu^{\#} .
\end{aligned}
$$

Hence, for $k>m_{0}$, we have

$$
\begin{aligned}
& \left|\int_{E} S_{k} f d \mu^{\#}-\mu^{\#}(E) \int_{\Omega^{*}} f d \mu^{\#}\right| \\
& \quad \leq\left|\int_{E} S_{k} f d \mu^{\#}-\int_{E_{p_{0}}} S_{k} f d \mu^{\#}\right|+\left(\mu^{\#}\left(E_{p_{0}}\right)-\mu^{\#}(E)\right)\left|\int_{\Omega^{*}} f d \mu^{\#}\right| \\
& \quad \leq \varepsilon+\varepsilon\|f\|_{1} .
\end{aligned}
$$

Hence $\lim _{k \rightarrow \infty} \int_{E} S_{k} f d \mu^{\#}=\mu^{\#}(E) \int_{\Omega^{*}} f d \mu^{\#}$ when $f \in L^{\infty}\left(\Omega^{\#}\right)$. Suppose now that $f$ is an arbitrary function in $L^{1}\left(\Omega^{\#}\right)$. Let $g \in L^{\infty}\left(\Omega^{\#}\right)$. Since $\left\|S_{k} g-S_{k} f\right\|_{1}=\|g-f\|_{1}$, we have

$$
\begin{aligned}
& \left|\int_{E} S_{k} f d \mu^{\#}-\mu^{\#}(E) \int_{\Omega^{*}} f d \mu^{\#}\right| \\
& \leq\left|\int_{E} S_{k} f d \mu^{\#}-\int_{E} S_{k} g d \mu^{\#}\right|+\left|\int_{E} S_{k} g d \mu^{*}-\mu^{\#}(E) \int_{\Omega^{*}} g d \mu^{\#}\right| \\
& \quad+\left|\int_{\Omega^{*}} f d \mu^{\#}-\int_{\Omega^{*}} g d \mu^{\#}\right| \\
& \quad \leq 2\|f-g\|_{1}+\left|\int_{E} S_{k} g d \mu^{\#}-\mu^{\#}(E) \int_{\Omega^{*}} g d \mu^{\#}\right| .
\end{aligned}
$$

Hence the desired result follows from the case $f \in L^{\infty}\left(\Omega^{\#}\right)$.

Proof of Theorem 4.2. Suppose that $\varphi$ is a function in $C_{c}^{\infty}\left(\widehat{\mathbf{R}}^{n}\right)$ such that

$$
\int_{\widehat{\mathbb{R}}^{n}} \varphi(\gamma) d e_{\gamma}^{\#} \mid L^{2}(D)=0 .
$$

To prove the theorem, it suffices to show that $\varphi$ vanishes on $\Gamma^{\prime}$. In view of Theorem 4.1, we only need to show that $\int_{\widehat{R}^{n}} \varphi(\gamma) d e_{\gamma}^{*}=0$. Let $(2 \pi)^{n / 2} \check{\varphi}$ be the inverse Fourier transform of $\varphi$, i.e., $\varphi(\gamma)=\int_{\mathbb{R}^{n}} \exp (i\langle t, \gamma\rangle) \check{\varphi}(t) d t$. Then for every $f \in L^{2}\left(\Omega^{\#}\right)$, 


$$
\chi_{D} \int_{\mathbf{R}^{n}} \check{\varphi}(t) u_{t}^{\#} f d t=\chi_{D} \int_{\widehat{\mathbf{R}}^{n}} \varphi(\gamma) d e_{\gamma}^{\#} f=\int_{\widehat{\mathbf{R}^{n}}} \varphi(\gamma) d e_{\gamma}^{\#} \chi_{D} f=0
$$

By the preceding lemma, for any $f, g \in L^{2}\left(\Omega^{\#}\right)$, we have

$$
\begin{gathered}
\mu^{\#}(D)\left\langle\int_{\widehat{\mathbf{R}}^{n}} \varphi(\gamma) d e_{\gamma}^{\#} f, g\right\rangle=\mu^{\#}(D)\left\langle\int_{\mathbf{R}^{n}} \check{\varphi}(t) f \circ \alpha_{t}^{\#} d t, g\right\rangle \\
=\int_{\mathbf{R}^{n}} \check{\varphi}(t) \lim _{k \rightarrow \infty}\left\langle\chi_{D}\left(S_{k} f\right) \circ \alpha_{t}^{\#}, S_{k} g\right\rangle d t .
\end{gathered}
$$

Here, we used the fact that $S_{k}\left(f \circ \alpha_{t}^{\#}\right)=\left(S_{k} f\right) \circ \alpha_{t}^{\#}$. By the dominated convergence theorem, we have

$$
\begin{gathered}
\mu^{\#}(D)\left\langle\int_{\widehat{\mathbf{R}}^{n}} \varphi(\gamma) d e_{\gamma}^{\#} f, g\right\rangle=\lim _{k \rightarrow \infty} \int_{\mathbf{R}^{n}} \check{\varphi}(t)\left\langle\chi_{D}\left(S_{k} f\right) \circ \alpha_{t}^{\#}, S_{k} g\right\rangle d t \\
=\lim _{k \rightarrow \infty}\left\langle\chi D \int_{\mathbf{R}^{n}} \check{\varphi}(t) u_{t}^{\#} S_{k} f d t, S_{k} g\right\rangle=0 .
\end{gathered}
$$

Therefore $\int_{\widehat{R}^{n}} \varphi(\gamma) d e_{\gamma}^{\#}=0$.

It follows from Theorem 4.2 that $\left\{\left(\Omega^{\#}, \mathscr{B}^{\#}, \mu^{\#}\right),\left\{\alpha_{t}^{\#}: t \in \mathbf{R}^{n}\right\}\right\}$ satisfies the condition that we imposed on our dynamical systems in $\S 1$. Therefore the theorems stated in $\S 2$ are valid for the pseudo-differential operators associated with this new dynamical system. Suppose now that $a$ is a function on $\Omega \times \widehat{\mathbf{R}}^{n}$ which belongs to $S^{m}$ for some $m \in \mathbf{R}$. That is, there is a set $\Lambda \in \mathscr{B}$ of measure 0 such that for any pair of multi-indices $\nu$ and $\beta$, there is a $C_{\nu \beta}>0$ such that

$$
\sup _{\omega \in \Omega \backslash \Lambda}\left|\partial_{x}^{\nu} \partial_{t}^{\beta} a\left(\alpha_{t}(\omega), x\right)\right| \leq C_{\nu \beta}(1+|x|)^{m-|\nu|} .
$$

Define

$$
a^{\#}\left(\left(\omega_{1}, \ldots, \omega_{k}, \ldots\right), x\right)=a\left(\omega_{1}, x\right)
$$

for $x \in \widehat{\mathbf{R}}^{n}$ and $\left(\omega_{1}, \ldots, \omega_{k}, \ldots\right) \in \Omega^{\#}=\Omega \times \cdots \times \Omega \times \cdots$. Then $a^{\#}$ belongs to the class $S^{m}$ on $\Omega^{\#} \times \widehat{\mathbf{R}}^{n}$. In exactly the same way we defined $A$ on $L^{2}\left(\Omega \times\left(\mathbf{R}^{n} / \Gamma\right)\right)$ and $A^{\gamma}$ on $L^{2}(\Omega)$, we can now define the pseudodifferential operators $A^{\#}$ on $L^{2}\left(\Omega^{\#} \times\left(\mathbf{R}^{n} / \Gamma\right)\right)$ and $A^{\# \gamma}$ on $L^{2}\left(\Omega^{\#}\right)$ using the symbol function $a^{\#}$. Therefore if we apply Theorems $1-5$ and 4.2 , we have the following theorems on the spectra of various pseudo-differential operators with $a^{\#}$ or $a$ as their symbol.

Theorem $1^{\#}$. (i) For every $\gamma \in \Gamma^{\prime}, \sigma_{l}\left(A^{\# \gamma}\right)=\sigma_{l}\left(A^{\#}\right)$.

(ii) The operator $A^{\#}$ is bounded if and only if $A^{\# \gamma}$ is bounded for every $\gamma \in \Gamma^{\prime}$.

(iii) In the case $A^{\#}$ is bounded, $\left\|A^{\#}\right\|=\left\|A^{\# \gamma}\right\|$ for every $\gamma \in \Gamma^{\prime}$.

(iv) In the case $A^{\#}$ is bounded, $\sigma\left(A^{\# \gamma}\right)=\sigma\left(A^{\#}\right)$ for every $\gamma \in \Gamma^{\prime}$.

Theorem $2^{\#}$. The following are equivalent:

(i) There is a $\gamma \in \Gamma^{\prime}$ such that $A^{\# \gamma}$ is a symmetric operator.

(ii) For every $\gamma \in \Gamma^{\prime}, A^{\# \gamma}$ is a symmetric operator.

(iii) The operator $A^{\#}$ is symmetric.

(iv) $A_{\omega}$ is symmetric for a.e. $\omega \in \Omega$.

Theorem $3^{\#}$. Suppose that the symbol function $a$ is elliptic and formally selfadjoint. Then $\sigma\left(A^{\# \gamma}\right)=\sigma\left(A^{\#}\right)$ for every $\gamma \in \Gamma^{\prime}$. 
Theorem $4^{\#}$. Suppose that the symbol function $a$ is elliptic and formally selfadjoint. Then:

(i) For a.e. $\omega \in \Omega, \sigma\left(A_{\omega}\right) \subset \sigma\left(A^{\#}\right)$.

(ii) If $A^{\#}$ is bounded, then so is $A_{\omega}$ and $\left\|A_{\omega}\right\| \leq\left\|A^{*}\right\|$ for a.e. $\omega \in \Omega$.

Theorem $\mathbf{5}^{\#}$. Suppose that $A^{\#}$ is selfadjoint. Let $B_{R}=\left\{\gamma \in \Gamma^{\prime}:|\gamma| \leq R\right\}$ for $R>0$. There is a positive Borel measure $V_{R}^{\#}$ on $\mathbf{R}$ such that

$$
\int_{B_{R}}\left\langle\left(A^{\# \gamma}-z\right)^{-1} 1,1\right\rangle d m(\gamma)=\int_{\mathbf{R}} \frac{1}{\lambda-z} d V_{R}^{\#}(\lambda) .
$$

Furthermore, $\sigma\left(A^{\#}\right)$ is the collection of $\lambda \in \mathbf{R}$ which satisfies the condition that for every $\varepsilon>0$ there is an $R=R(\varepsilon)>0$ such that $V_{R}^{\#}((\lambda-\varepsilon, \lambda+\varepsilon))>0$.

\section{REFERENCES}

1. W. Arveson, On the group of automorphisms of operator algebras, J. Funct. Anal. 15 (1974), 217-243.

2. R. Biktashev and A. Mishchenko, Spectra of elliptic unbounded pseudodifferential operators over $C^{\infty}$-algebras, Moscow Univ. Math. Bull. 35 (1980), 59-62.

3. M. Burnat, Die Spektraldarstellung einiger Differentialoperatoren mit periodischen Koeffizienten im Raume der fastperiodischen Funktionen, Studia Math. 25 (1964/65), 33-64.

4. L. Coburn, R. Douglas, D. Shaeffer, and I. Singer, On the $C^{*}$-algebras of operators on a half-space. II, Index theory, Inst. Haute Études Sci. Publ. Math. 40 (1971), 69-79.

5. L. Coburn, R. Moyer, and I. Singer, $C^{*}$-algebras of almost periodic pseudo-differential operators, Acta Math. 130 (1973), 279-307.

6. P. Dedik and M. Shubin, Random pseudo-differential operators and the stabilization of solutions of parabolic equations with random coefficients, Math. USSR-Sb. 41 (1982), 33-52.

7. R. Douglas, On the $C^{*}$-algebra of a one-parameter semi-group of isometries, Acta Math. 128 (1972), 143-151.

8. F. Forelli, Analytic and quasi-invariant measures, Acta Math. 118 (1967), 33-59.

9. J. Kaminker and J. Xia, The spectrum of operators elliptic along orbits of $\mathbf{R}^{n}$ actions, Comm. Math. Phys. 110 (1987), 427-438.

10. S. Kozlov and M. Shubin, On the coincidence of the spectra of random elliptic operators, Math. USSR-Sb. 51 (1985), 455-471.

11. R. Loebl and P. Muhly, Analy'ticity and flows on von Neumann algebras, J. Funct. Anal. 29 (1978), 214-252.

12. P. Muhly, I. Putnam, and J. Sia, On the $K$-theory of some $C^{*}$-algebras of Toeplitz and singular integral operators, J. Funct. Anal. 110 (1992), 161-225.

13. P. Muhly and J. Xia, $C^{*}$-algebras of singular integral operators and Toeplitz operators associated with n-dimensional flows, Internat. J. Math. 4 (1992), 525-579.

14. A. Pankov, On the theory of almost periodic pseudo-differential operators, Ukrainian Math. J. 33 (1981), 469-472.

15. M. Shubin, The essential self-adjointness of uniformly hypoelliptic operators, Moscow Univ. Math. Bull. 30 (1975), 147-150.

16. Theorems on the coincidence of the spectra of a pseudo-differential operator in the spaces $L^{2}\left(\mathbf{R}^{n}\right)$ and $B^{2}\left(\mathbf{R}^{n}\right)$, Siberian Math. J. 17 (1976), 158-170.

Department of Mathematics, State University of New York at Buffalo, Buffalo, NEW YORK 14214 